Original Paper

\title{
Computational Investigations of Impingement Heat Transfer on an Effused Concave Surface
}

\author{
M. Ashok Kumar and Bhamidi V.S.S.S. Prasad \\ Thermal Turbomachines Laboratory, Department of Mechanical Engineering, \\ Indian Institute of Technology Madras, Chennai 600036, India. \\ lalgudiashok@gmail.com, prasad@iitm.ac.in
}

\begin{abstract}
A computational study is reported on flow and heat transfer characteristics from five rows of circular air jets impinging on a concave surface with four rows of effusion holes. The effects of exit configurations of spent air and the arrangement of jet orifices and effusion holes for a jet Reynolds number of 7500 is investigated. In all, eight cases are studied and a good qualitative correlation is found among their flow patterns, pressure variations and heat transfer distributions
\end{abstract}

Keywords: Gas turbine blade cooling, Impingement cooling, Film holes, CFD

\section{Introduction}

This Guide The leading edge of a modern aircraft gas turbine engine is typically protected by a combined jet and effusion cooling, which offer a high heat transfer coefficient. The coolant jet impinges on the interior surface of the leading edge and exits through the effusion or film holes and/or through sideways depending on the design configurations. The flow features and the values of heat transfer coefficient in the vicinity of leading edge are therefore significantly influenced by the effusion as well as the exit configuration schemes of the coolant.

Although a large number of research investigations are carried out on the impingement or film cooling surfaces in isolation, very few studies are available on the combined cooling. Hollworth and co-workers $[1,2]$ reported perhaps the first investigations on the air jets impinging on a flat surface, with the spent fluid removed through film holes from the target surface. The jet and the film holes had the same diameter, whilst the spacing of the jet holes was twice that of the film holes. They found an increase of about $35 \%$ in heat transfer rate with an array of staggered film holes in comparison with the case without film holes. They attributed this increase to boundary layer suction in the vicinity of the film holes. Cho and Rhee [3] conducted mass transfer experiments and reported heat/mass transfer results for multiple jets impinging on the flat surface and exiting through the effusion holes. They found a peak in this Nusselt number between the two jets, close to the up-wash zone. They reasoned that a pair of secondary vortices formed a secondary stagnation zone and hence this Nusselt number peak. However, they were unable to capture the secondary vortices and the secondary stagnation zone with this RSM model used for prediction.

By conducting experimental investigations on flat surface with effusion holes for three cases of exit flow configurations (i) flow exits in the same direction as entry (ii) flow exits in an opposite direction to the entry and (iii) flow exits in both the directions, Ekkad et al [4] noted significant variations among heat transfer distributions for different cases. They attributed these variations to the difference in cross flow effect caused due to different exit configuration. Rhee and co-workers [5, 6] supported these arguments by observing highly non-uniform heat transfer coefficients noticed due to strong effects of cross flow and reentrainment of spent air. However, they achieved uniform distribution and enhancement of transport rate with the addition of film holes.

Whilst all the above studies are on flat surfaces, Metzger et al. [7] showed that the circular jet impinging on a concave surface would produce higher heat transfer coefficient than when the comparable two-dimensional jets impinge on a plane surface. Thomann [8] quantified the heat transfer to be about twenty percent higher on the concave surface than on the flat surface. Tabakoff and Clevenger [9] conducted experimental investigations for multiple jets impinging on the concave surface for two exit configurations: (i) side edges are blocked and both top edges are open and (ii) side edges and one of the top edges are blocked. They found that by closing one side the heat transfer performance did not alter much. 
All the above mentioned studies on the concave surfaces are without effusion holes. By conducting experiments when a single row of jet impinges on a concave surface with extraction of film coolant, Bunker and Metzger [10, 11] noted that the flow extraction showed only a small influence on heat transfer. They also pointed out that the jets inline with film holes would yield higher heat transfer coefficient than when they are in staggered configuration. However these heat transfer data showed significant differences among various investigators. For instance, Taslim et al [12] concluded that the presence of film holes enhanced the internal impingement heat transfer coefficient significantly. However, their numerical estimates using k- $\varepsilon$ model of underpredicted average heat transfer values by as much as thirty percent in the leading edge region. They suggested the need of using a better turbulence model. Ramakumar and Prasad [13, 14] demonstrated that k- $\omega$ SST model predicts the heat transfer characteristics more closely with the experimental data on concave surface with multiple impinging jets. However, they have not tested their method with effusion/film holes.

In view of the foregoing literature, computational investigations in the paper are conducted for concave surface with multiple rows of impinging jets and effusion holes for (a) staggered and inline arrangement of effusion holes and (b) four numbers of exit configurations for a height to diameter ratio of unity. In all, eight cases are solved and the numerical results are reported for a jet Reynolds number of 7500 .

\section{Methodology}

\subsection{Physical and Computational models}

The physical model with $5 \times 4$ rows of round jets impinging on the concave target surface is considered for the present study (Fig. 1). The target surface contains $4 \times 4$ rows of effusion holes. This physical model closely simulates the impingement cooling of a gas turbine blade leading edge with effusion holes. The air jets, after impinging on the target surface, exit through the effusion holes, and optionally (four different cases) along the edges of the target surface. Although the actual leading edge of the turbine vane is not of circular shape, the circular profile being generic in nature is considered to be the best model for the purpose of characterizing the geometric parameters.

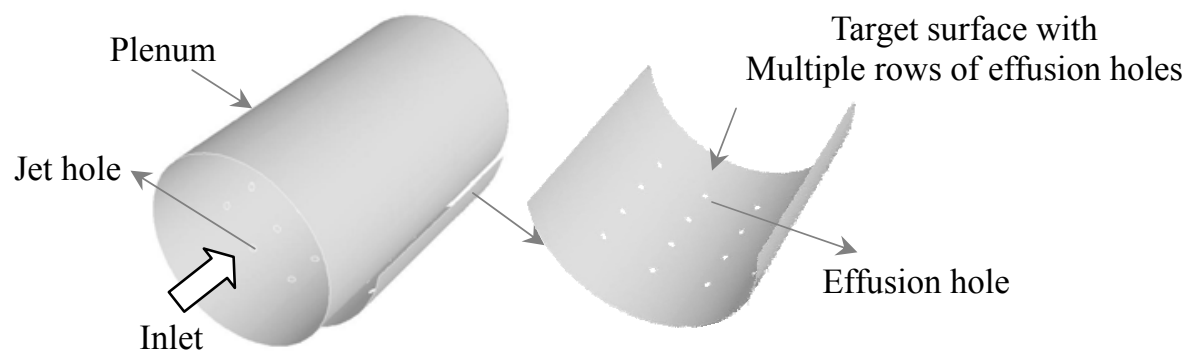

Fig. 1 Physical model

The computational domain closely mimics the physical model, which consists of a plenum cylinder with jet orifices and a concave target surface with effusion holes. The jet orifices are positioned to obtain an inline or a staggered arrangement with respect to the effusion holes. For the purpose of computations, a specified mass flow condition is imposed at the inlet. A turbulence intensity of 5\% is chosen at the inlet fluid. Ambient air of constant temperature at $308 \mathrm{~K}$ is specified as the inlet fluid. A constant wall temperature of $338 \mathrm{~K}$ (so that temperature difference is $30 \mathrm{~K}$ ) is applied to the target surface. On all other solid surfaces such as plenum cylinder and jet orifices, the adiabatic wall condition is imposed.

Table 1 Parameters investigated

\begin{tabular}{|c|c|}
\hline Parameters & Values \\
\hline Number of jets in an row & 4 \\
\hline Number of rows of jet (longitudinal) & 4 \\
\hline Number of rows of jet (circumferential) & 5 \\
\hline Number of effusion holes in a row & 4 \\
\hline Number of rows of effusion holes & 4 \\
\hline $\begin{array}{l}\text { Arrangement of jet orifices with reference to } \\
\text { effusion hole }\end{array}$ & staggered, inline \\
\hline Diameter of jet and effusion holes & $5 \mathrm{~mm}$ \\
\hline Spacing between jet holes and effusion holes, c & $27 \mathrm{~mm}$ \\
\hline $\begin{array}{l}\text { Spent air exit configurations } \\
\text { (refer Fig. 5ii for details) }\end{array}$ & $\begin{array}{l}\text { Case-A (all edges are open) } \\
\text { Case-B (two edges are open) } \\
\text { Case-C (one edge is open) } \\
\text { Case-D (all edges are closed) }\end{array}$ \\
\hline Jet Reynolds number $=4 \mathrm{~m} /(\pi \mathrm{D} \mu)$ & 7500 \\
\hline
\end{tabular}


Constant pressure outlet condition is applied on all outlet boundaries which include exit of all the effusion holes and the selected edges of the concave surface. Atmospheric pressure and temperature of $308 \mathrm{~K}$ are applied at these outlets. A typical computational domain and the corresponding mesh and the input conditions are shown in Figs 2 and 3 respectively. The near ambient conditions are chosen as the input conditions because the data from the present computations can be compared with the laboratory test data, the experiment which will be published separately at a later date.

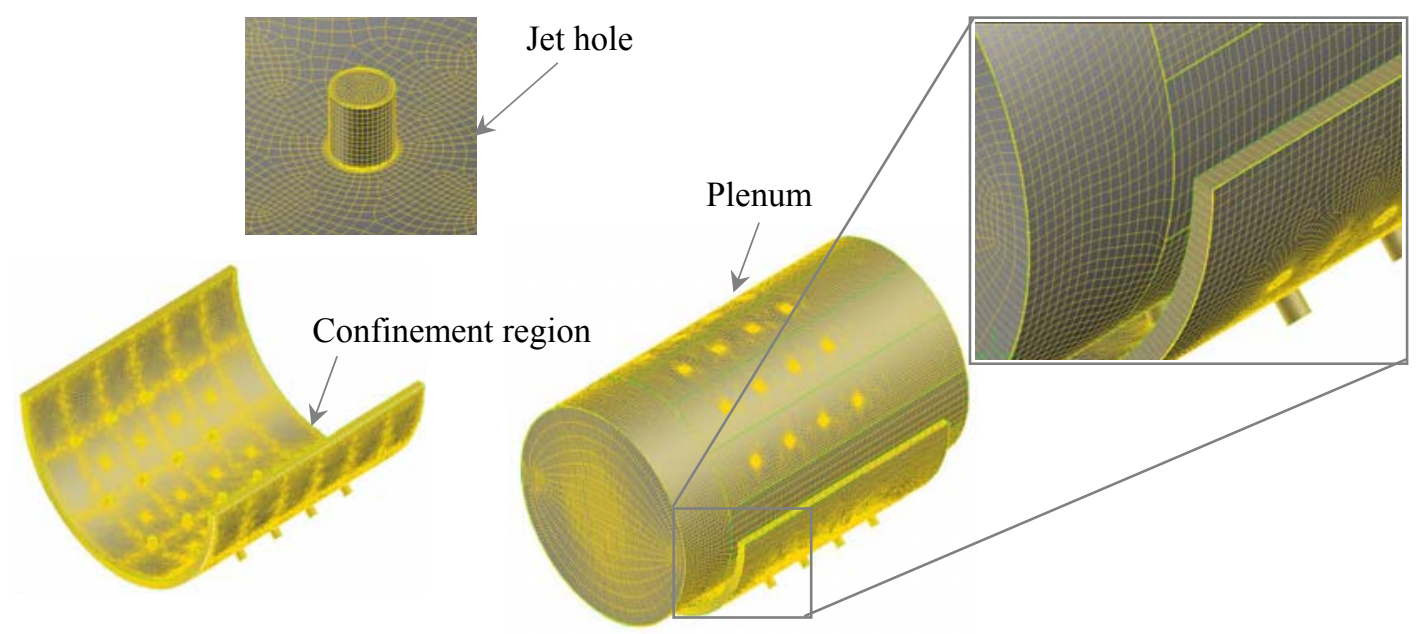

Fig. 2 Computational domain with mesh used

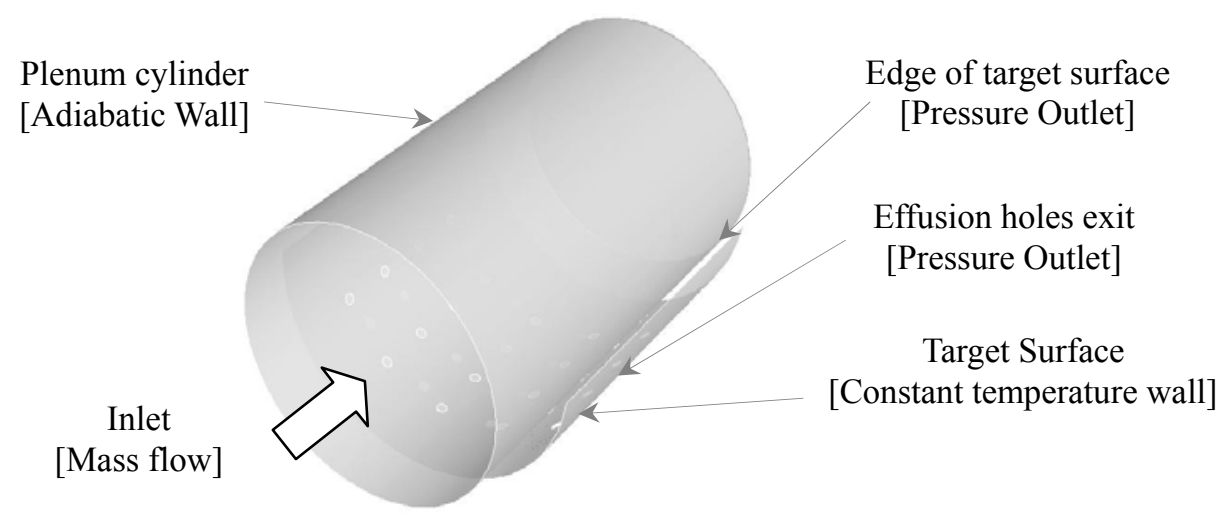

Fig. 3 Input conditions

\subsection{Governing equations}

The governing equations used for simulations are the Reynolds averaged continuity, momentum and the energy equations along with the equations for modeling the turbulence quantities. The assumptions made in formulating these equations for the present investigations are, steady, incompressible flow with constant properties and without viscous dissipation and buoyancy effects. The equations are as follows,

Continuity equation:

$$
\nabla \cdot(\rho u)=0
$$

Momentum equation:

$$
(u \cdot \nabla) u-\frac{1}{\rho} \nabla P+v \nabla^{2} u=0
$$

Energy equation is given by: $\rho c_{p}\left(\frac{\partial T}{\partial t}+u \cdot \nabla T\right)-k \nabla^{2} T=0$

TKE equation:

$$
\frac{\partial}{\partial t}(\rho \kappa)+\frac{\partial}{\partial x_{i}}\left(\rho \kappa u_{i}\right)=\frac{\partial}{\partial x_{j}}\left(\Gamma_{\kappa} \frac{\partial \kappa}{\partial x_{j}}\right)+G_{\kappa}-Y_{\kappa}+S_{\kappa}
$$


SDR equation:

$\frac{\partial}{\partial t}(\rho \omega)+\frac{\partial}{\partial x_{i}}\left(\rho \omega u_{i}\right)=\frac{\partial}{\partial x_{j}}\left(\Gamma \omega \frac{\partial \omega}{\partial x_{j}}\right)+G \omega-Y \omega+D \omega+S \omega$

Where, $\Gamma \kappa=\mu+\frac{\mu_{t}}{\sigma_{\kappa}}, \Gamma \omega=\mu+\frac{\mu_{t}}{\sigma_{\kappa}}, \sigma_{\kappa, 1}=1.176, \sigma_{\omega, 1}=2.0, \sigma_{\kappa, 2}=1.0, \sigma_{\omega, 2}=1.168$

\subsection{Numerical solution}

A finite volume based solver Fluent 6.3 is used for solving the governing equations and turbulence model. Flow is considered incompressible and constant properties are used because of the small variation in temperature and pressure. Second order upwind scheme is used for discretisation and SIMPLE algorithm is used for Pressure-velocity coupling. The solution is considered as converged when the residuals fall below $10^{-4}$ for momentum, continuity and turbulence equations and $10^{-8}$ for energy equation. The average Nusselt number along stagnation line is continuously monitored so that there will be no change in the value for consecutive 300 iterations even after the above residuals are reached.

\subsection{Validation}

It is already concluded by the authors that the SST k- $\omega$ turbulence model predicts well the flow and heat transfer characteristics for multiple impinging jets with effusion holes [15]. However, it is for jets impinging on flat surface with effusion holes. In order to find the validity of using the SST k- $\omega$ turbulence model for predicting the flow and heat transfer characteristics of jet impinging on a curved surface, a case with single jet impinging on concave surface is selected. The computational model selected for validating the present numerical methodology mimics the experimental set-up of Lee et al. [16] so that the computational results can be compared with the experimental data [16]. The pressure coefficient and the Nusselt number distribution with SST k- $\omega$ turbulence model along the curvature (refer Fig. 3) shows that there is a good agreement between the computational results and the experimental data [16]. Hence the present computational methodology with SST k- $\omega$ turbulence model can be well taken as validated for impinging jet on curved surface.

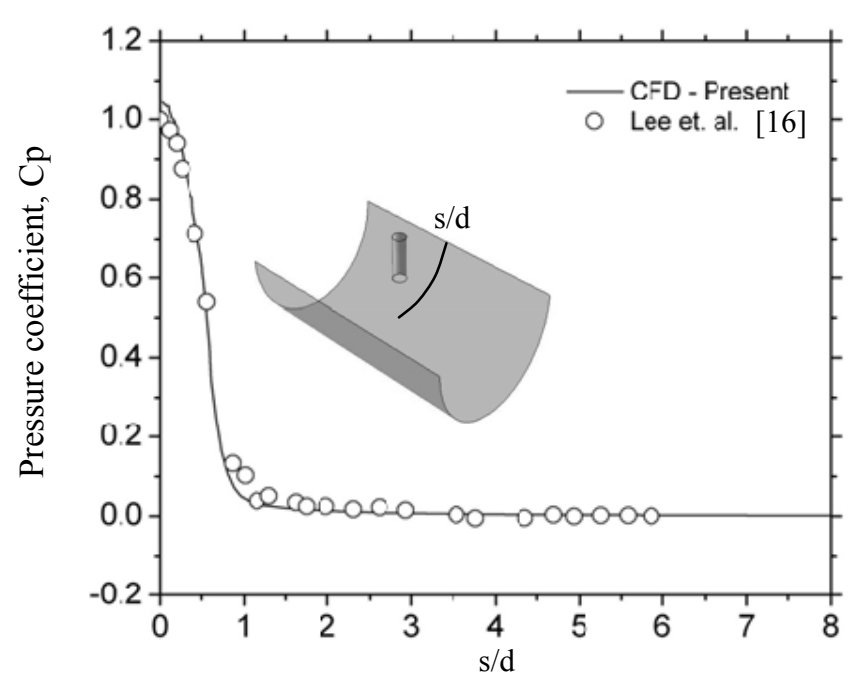

(a) Pressure coefficient distribution

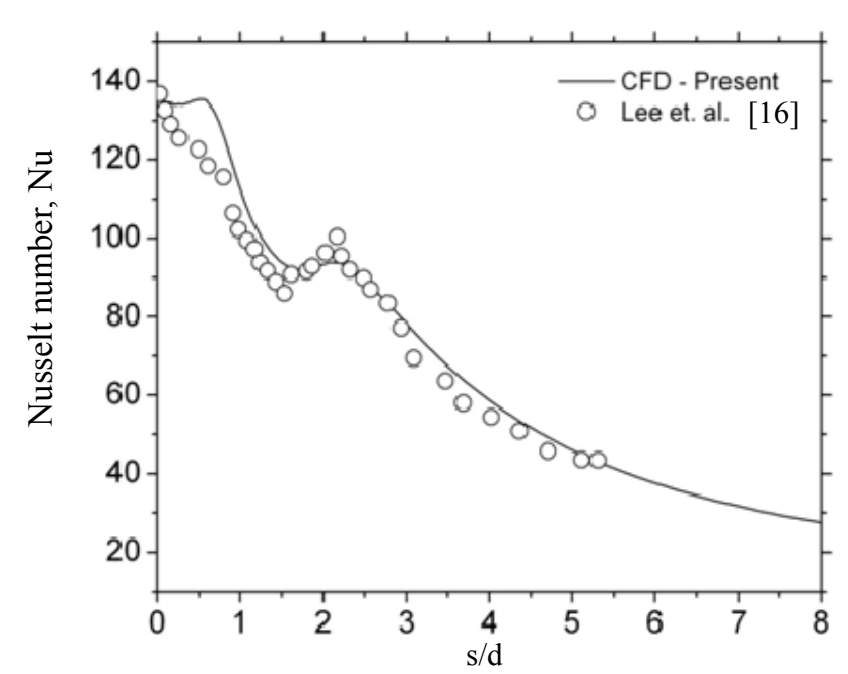

(b) Nusselt number distribution

Fig. 4 Validation of present methodology

\section{Results and Discussion}

\subsection{Parameters investigated}

The parameters investigated in the present study include (i) inline and staggered arrangements of jet orifices with respect to effusion holes (ii) four spent air exit configurations (A, B, C and D), refer Fig. 5. The parameters which are maintained constant are: Reynolds number at 7500 , jet orifice to effusion hole diameter ratio at unity, orifice thickness to diameter (t/D) at unity and pitch to diameter ratio (c/D) for jet and effusion holes at 5.4. Details of the parameters investigated are presented in Table-1 and Fig. 5. The path lines and the pressure and heat transfer distributions are plotted on the selected planes along lines ' $\mathrm{L} 1$ ' and ' $\mathrm{L} 2$ ' and curve $\mathrm{S}$, as shown in Fig. 6. 


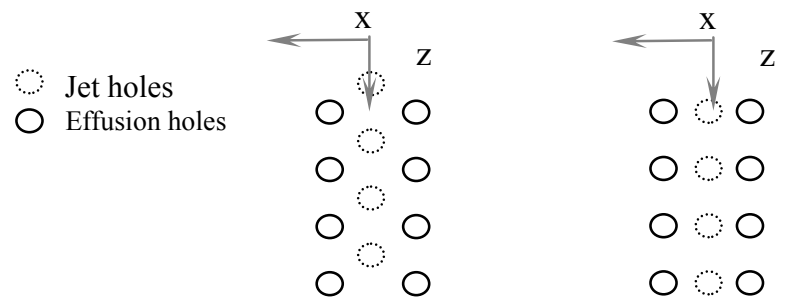

$\begin{array}{ll}\text { (a) Staggered (s) } & \text { (b) Inline (i) }\end{array}$

(i) Details of arrangement of jet and effusion holes

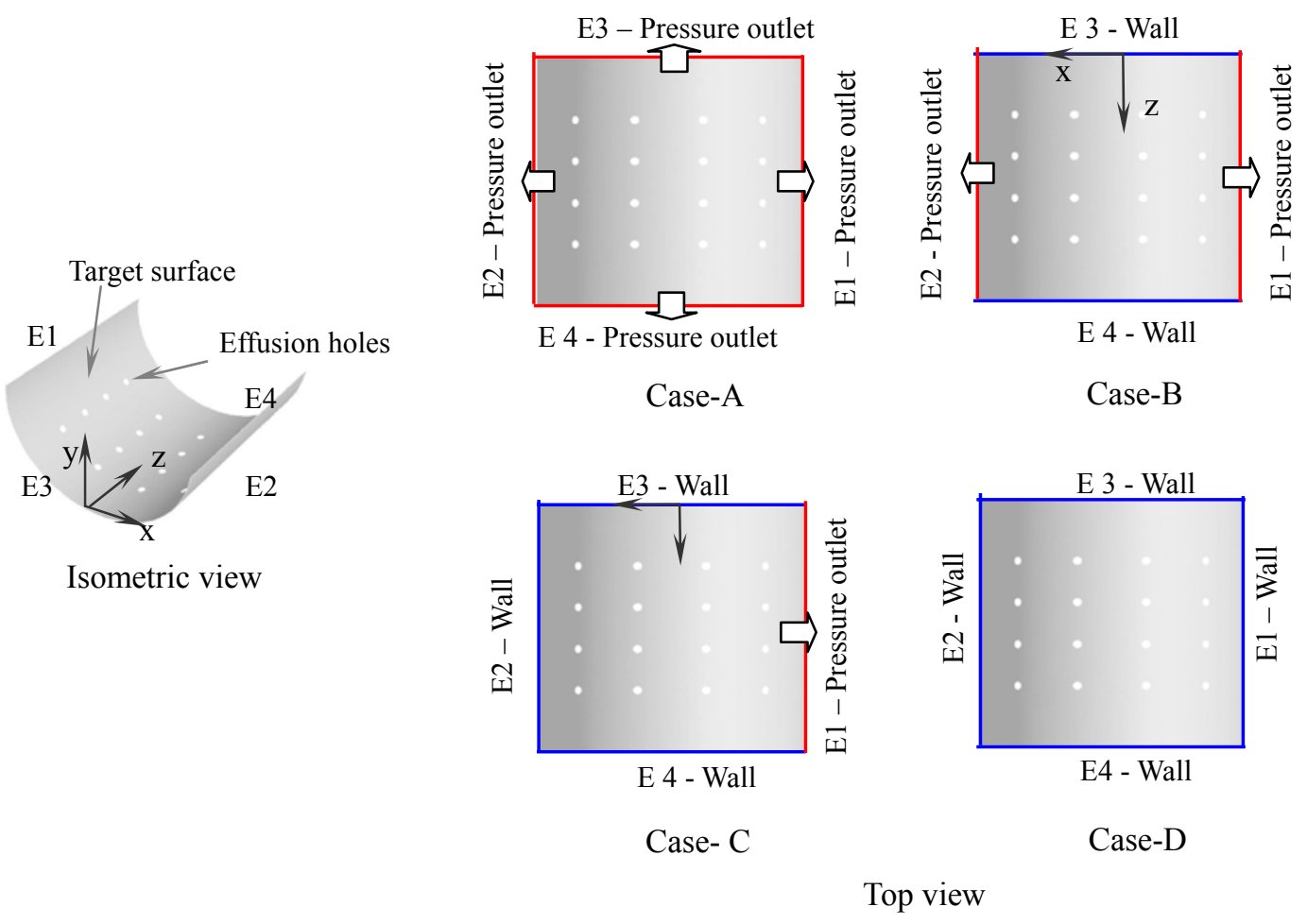

(ii) Spent air exit configurations

Fig. 5 Parameters investigated

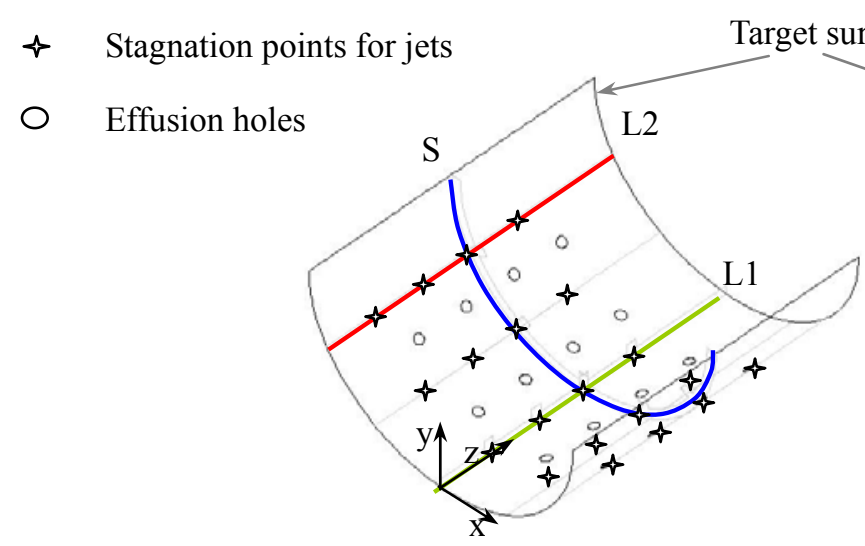

(a) Isometric view

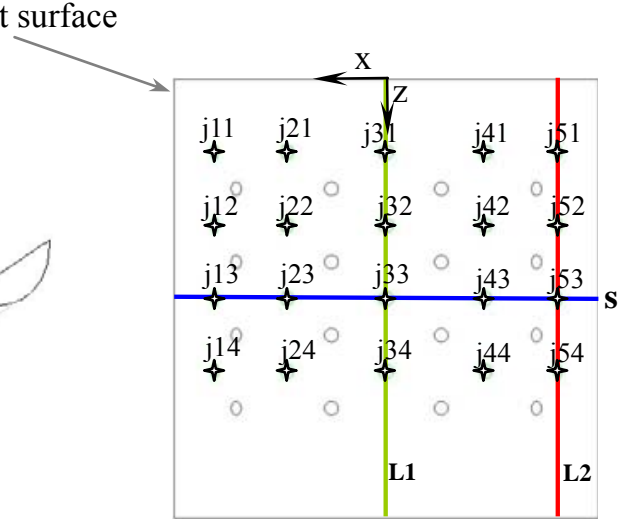

(b) Top view

Fig. 6 Lines and planes selected for plotting the results 


\subsection{Flow characteristics}

As the air jet impinges on the concave target surface it forms different patterns of vortex pair and exits along edges of the concave surface and through the effusion holes. The percent of air exiting through the effusion holes depends on the spent air exit configurations (A to D). These features are presented in Table 2. The reason for such variation in flow rates through the effusion holes is due to the variation in exit and vortex patterns for different configurations. These are discussed in the following sections.

Table 2 Percentage of air exits through effusion holes

\begin{tabular}{|c|c|c|}
\hline & Staggered (s) & Inline (i) \\
\hline Case-A & 5.7 & 2.0 \\
\hline Case-B & 13.3 & 14.1 \\
\hline Case-C & 25.1 & 27.8 \\
\hline Case-D & 100 & 100 \\
\hline
\end{tabular}

\subsubsection{Exit configuration 'A' with staggered hole arrangement (Case - As)}

Figure 7 shows the jet32 interacting with jet33 in the plane along 'L1' for Case-As. As the jet impinges on the target surface, it forms the primary stagnation zone, followed by wall jet region. As the neighboring wall jets interact with each other, large scale primary vortices (PV) are formed and the boundary layer of wall jet gets separated from the target surface. Small scale secondary vortices (SV) are thus created. It is noticed from Fig. 8(a) that the secondary vortices (formed for jet 31 interacting with jet 32 and for jet 33 interacting with jet 34) have shifted towards the open exits. It shows that the majority of fluid (about $94.3 \%$ ) exits through the edge of the concave surface and only $5.7 \%$ exits through the effusion holes. However, this shift is very small (Fig. $8 \mathrm{~b}$ ) along the line 'L2' as it is near the open edge. All the features such as formation of the primary vortices, up-wash region and secondary vortices are noticed in plane along ' $\mathrm{S}$ '. Figure $8 \mathrm{c}$ shows the path lines plotted along the curve ' $\mathrm{S}$ '. It is observed that the primary vortex length is smaller when plotted along the curve ' $\mathrm{S}$ '. The influence of effusion holes is not noticeable as very little flow takes place through these.

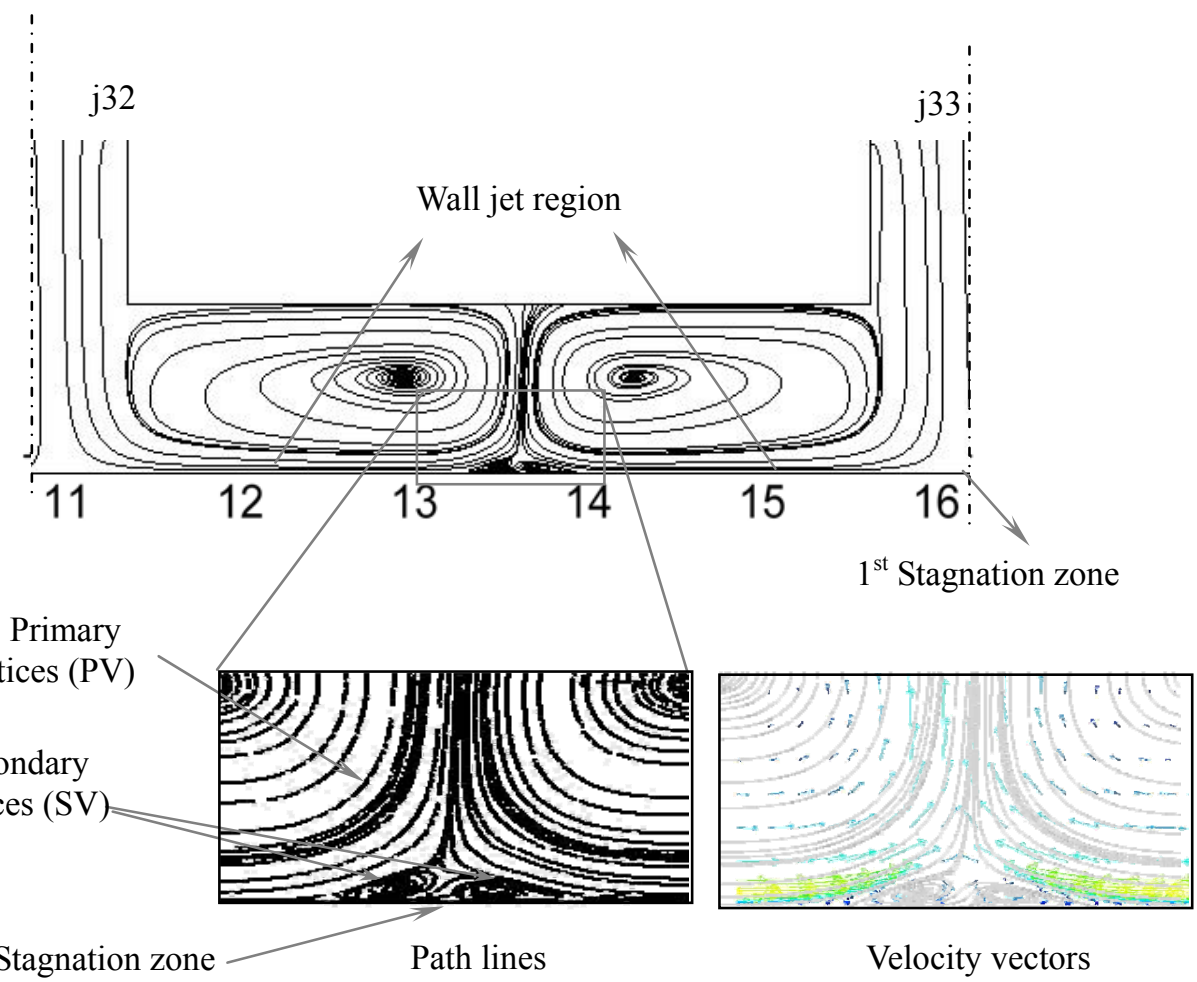

Fig. 7 Jet 32 interacting with jet 33 along planes L3 for Case-As 


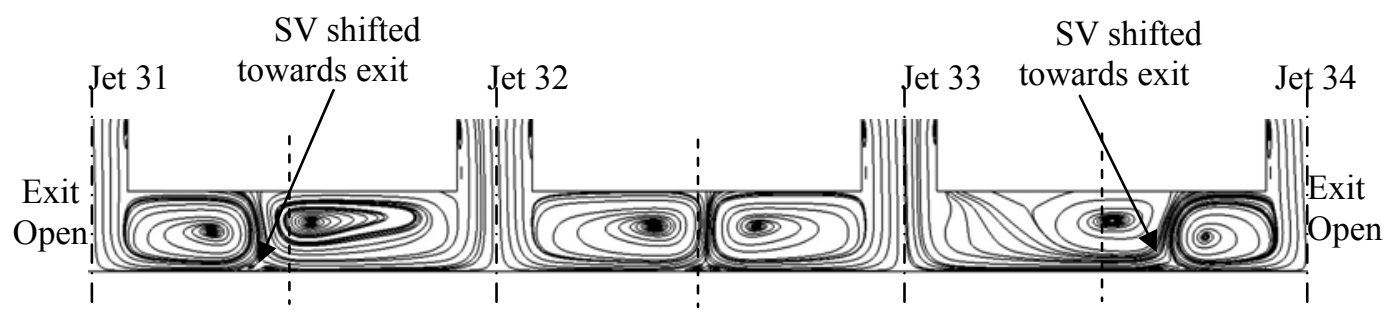

(a) Path lines in plane along L1 showing the shifting of secondary vortices towards the edge

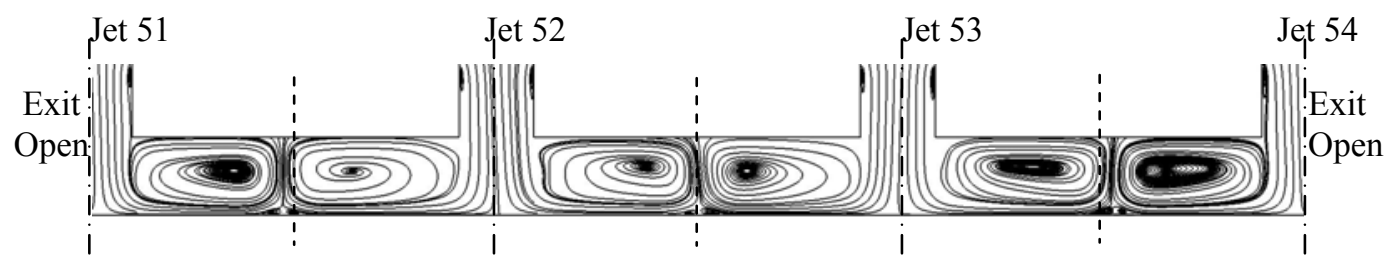

(b) plane along L2

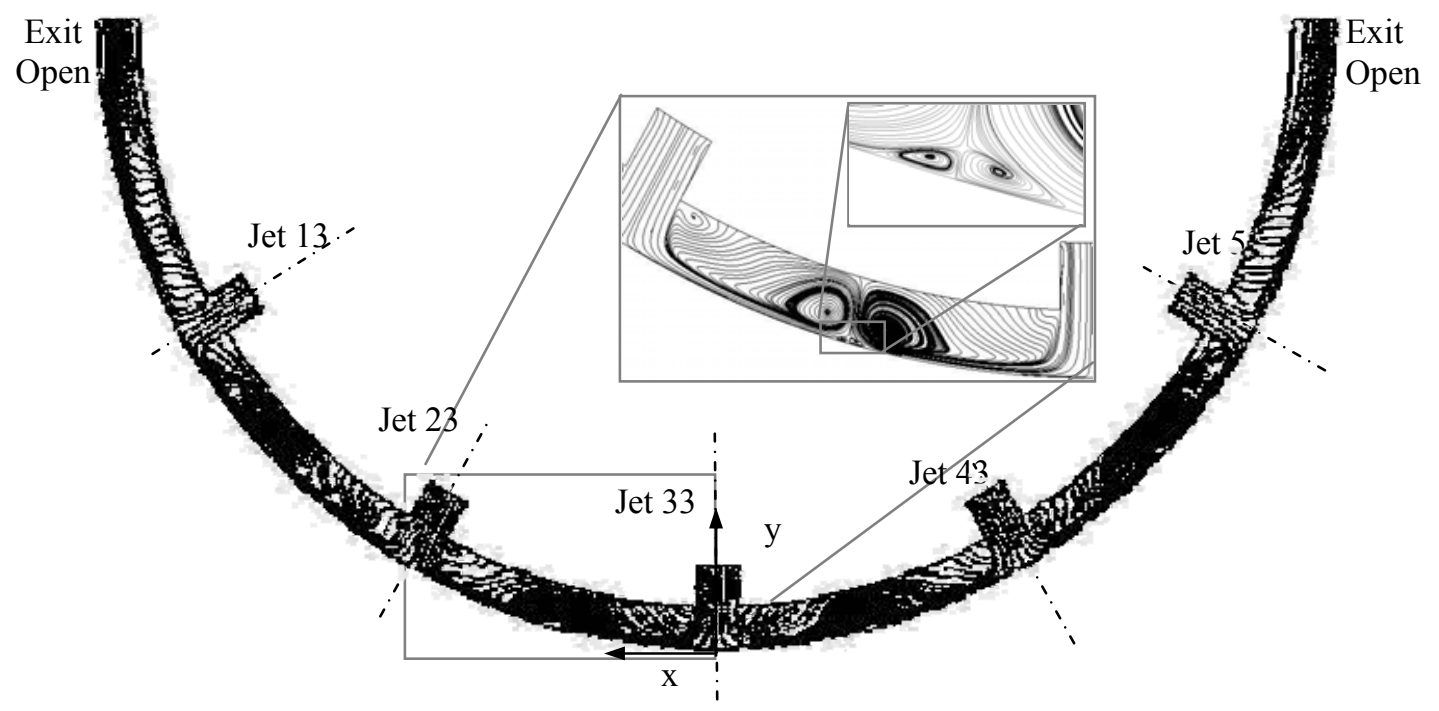

(c) plane along $\mathrm{S}$

Fig. 8 Path lines plotted for Case-As

\subsubsection{Exit configuration 'A' with inline hole arrangement (Case-Ai)}

The flow features observed for inline case along the planes 'L1' and 'L2' (Fig. 9a and b) are similar to those noticed for case As. However, as the effusion holes are partly occupied in the secondary stagnation zone along the curvature 'S'. Secondary vortices are not observed (Fig. 9c). Eddies are also seen to have formed in the effusion holes. These eddies block the flow through the effusion holes. Hence the percentage of air bleeding through the effusion hole is reduced a substantially low value of for inline arrangement of $2 \%$ when the effusion holes are arranged in-line, for the present (A) exit configuration. 


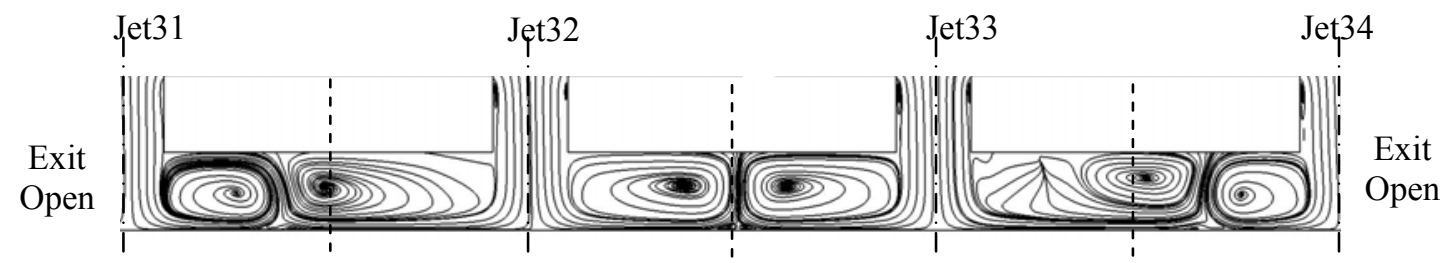

(a) plane along L1

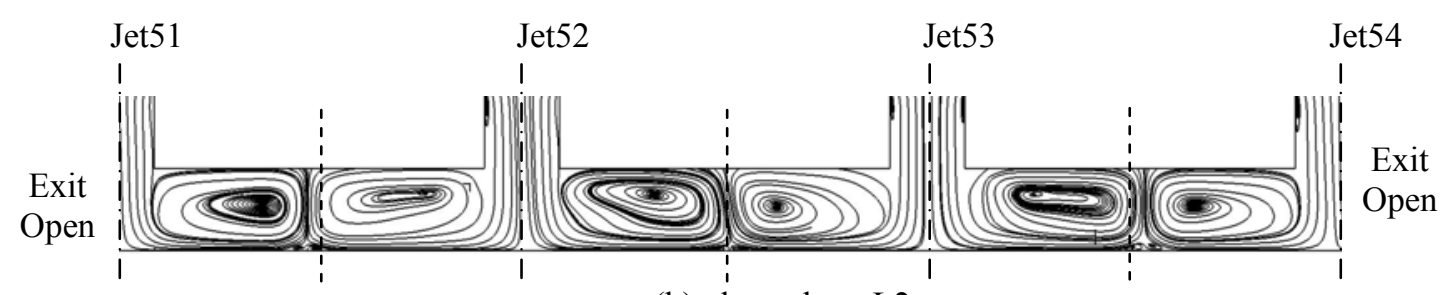

(b) plane along L2

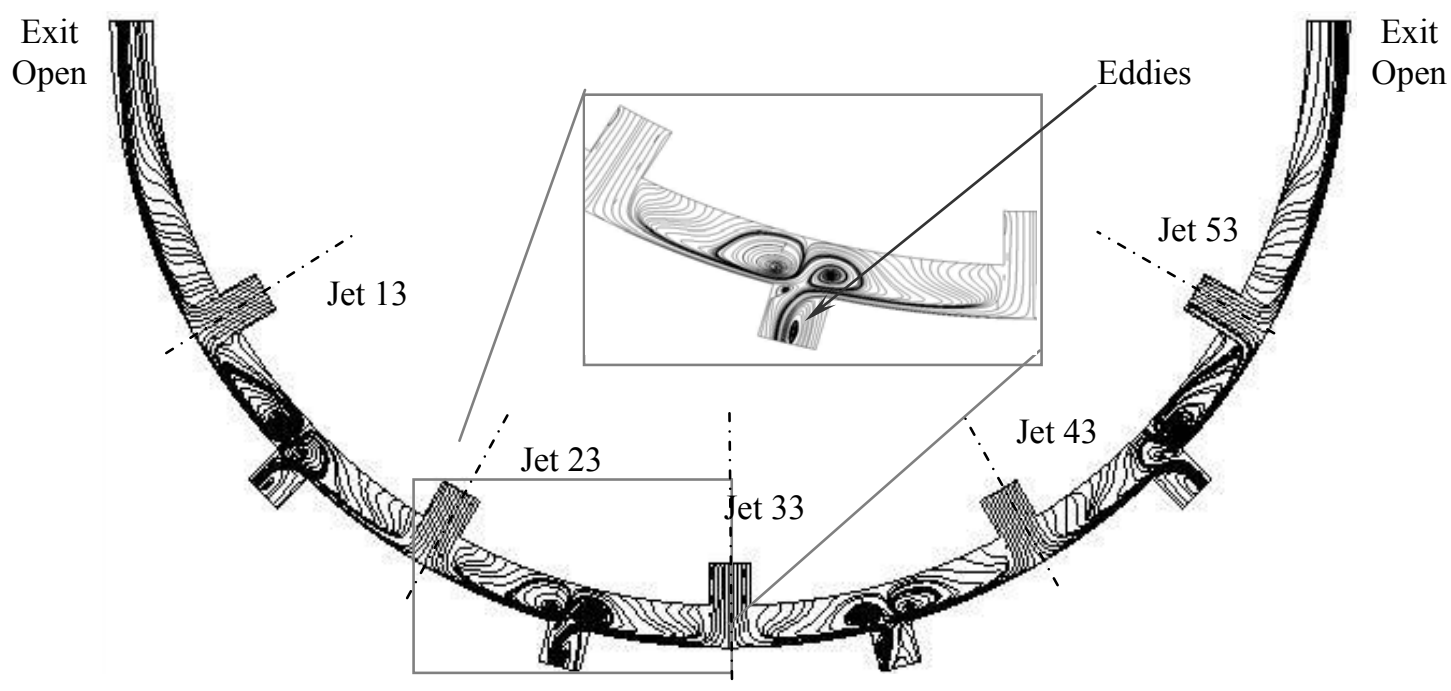

(c) plane along $\mathrm{S}$

Fig. 9 Path lines plotted for Case-Ai

\subsubsection{Exit configuration ' $B$ ' with staggered hole arrangement (Case - Bs)}

Due to the edge closure of edge E1 and E2 in Case-B, there will be an increase in internal cross flow along the curvature of the concave target surface which leads to the increase in the discharge air through the effusion holes the percent of air exiting through the effusion holes increases to about $13 \%$. Path lines plotted along the curve 'S' (Fig. 10) show the jet interactions and then formation of primary and secondary vortices when jet 33 interacting with jet 23 and jet 43 . However, due to the increased cross flow, there is a clear shift in the PV cells, formed between the jets (13 and 23) and the jets (43 and 53). 


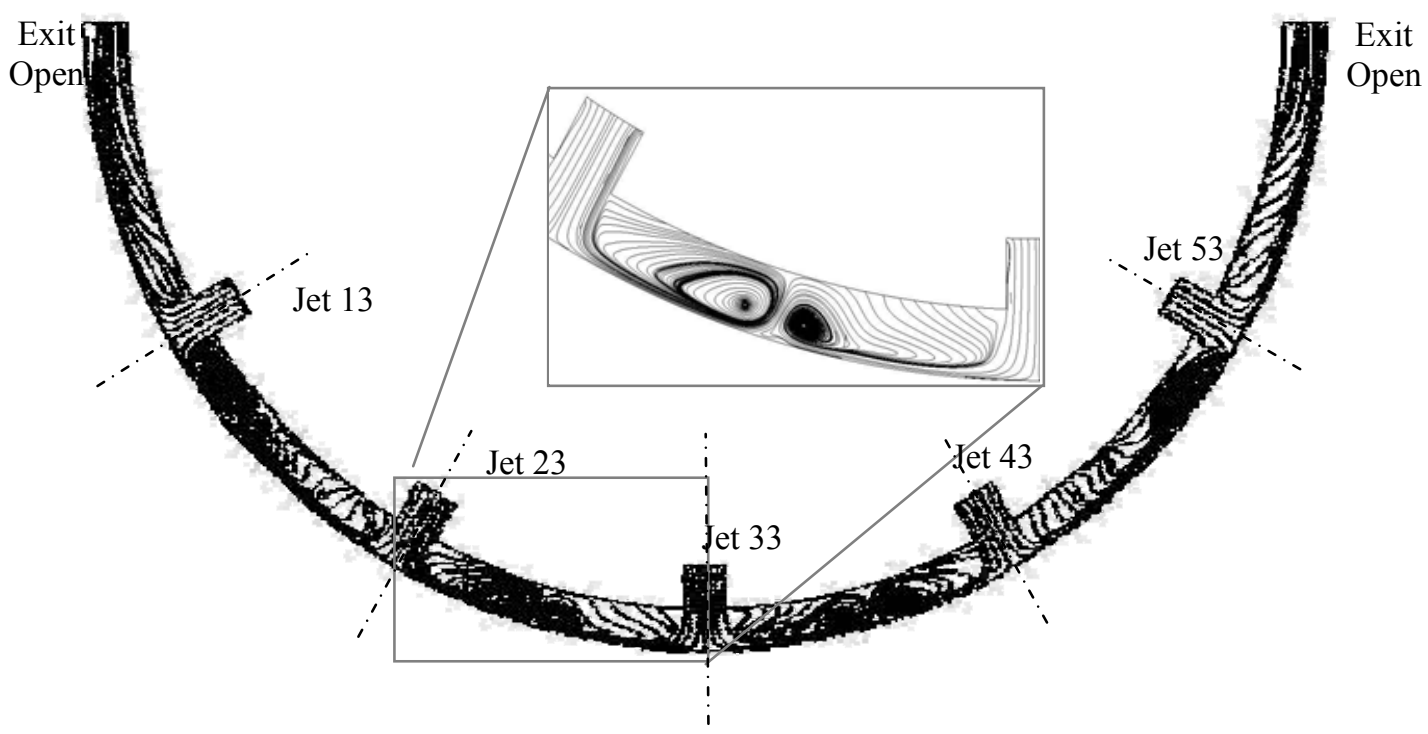

Fig. 10 Path lines plotted for Case-Bs

\subsubsection{Exit configuration ' $B$ ' with inline hole arrangement (Case - Bi)}

For the inline arrangement (Case-Bi), along 'S' (Fig. 11), the percentage of spent air exit through the effusion holes increases to about $14 \%$, due to reduced blockage in the effusion holes. The strength of the PV and SV cells is considerably reduced in the vicinity of the effusion holes.

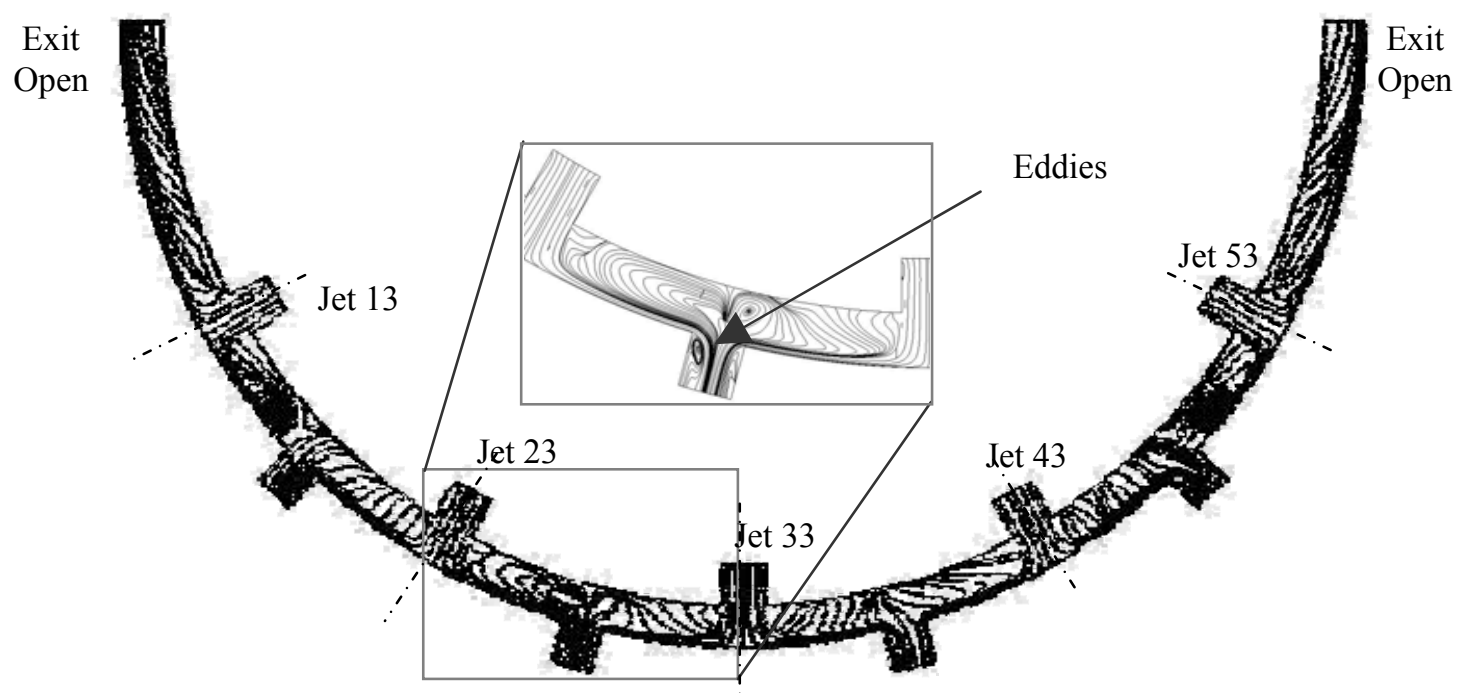

Fig. 11 Path lines plotted for Case-Bi

\subsubsection{Exit configuration ' $C$ ' with staggered hole arrangement (Case - Cs)}

For Case-Cs, where only one edge-E1 is open while other exits are closed, the secondary vortices are not clearly observed in plane passing through 'L2' (Fig. 12a), as the exiting flow behaves like a wash-away cross flow that weakens the jet interaction, preventing the formation of up-wash and secondary vortices. For the same reason, along the plane ' $\mathrm{S}$ ' (Fig. 12b), the primary vortices formed are pushed towards the open edge. This exit closure configuration increases the percentage of air exiting through the effusion holes further to about $25 \%$. 


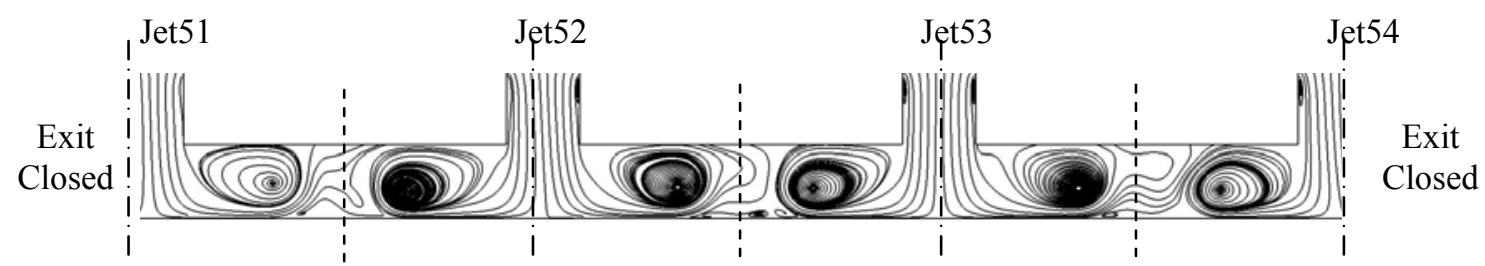

(a) plane along L2

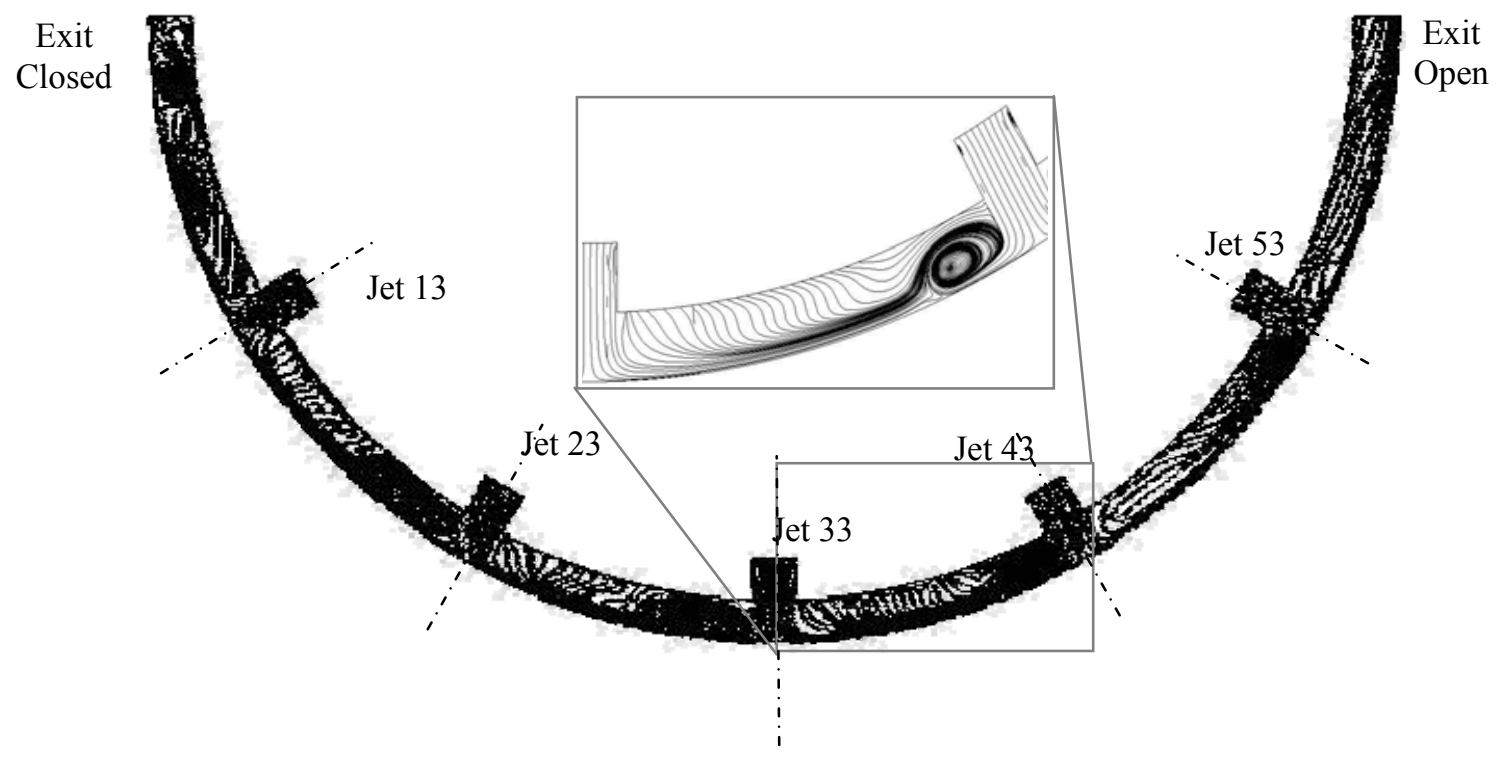

(b) along curve $\mathrm{S}$

Fig. 12 Path lines plotted for Case-Cs

\subsubsection{Exit configuration ' $C$ 'with inline hole arrangement (Case - Ci)}

When the holes are aligned, cross flow effects are reduced and the blockage in the effusion holes further reduced. These contribute to the increase in percent of air exit through the effusion holes to about $28 \%$ which leads to reduction in the cross flow. The jet interactions are not observed along 'S' (Fig. 13) due to increase in suction.

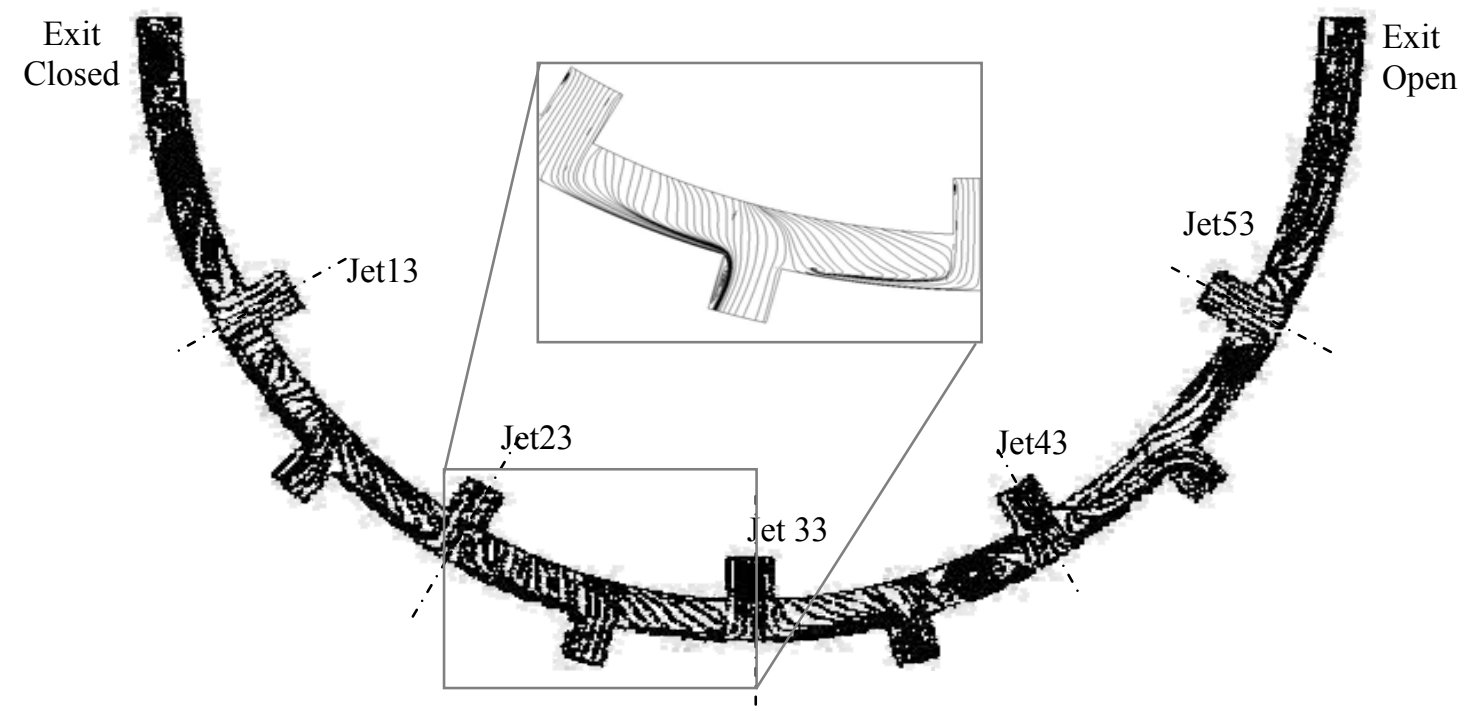

Fig. 13 Path lines plotted for Case-Cs 


\subsubsection{Exit configuration 'D'with staggered hole arrangement (Case - Ds)}

The jet interactions with the formation of PV and SV in all the planes are clearly observed for all the jets due to substantial reduction in internal cross flow (Figs. $14 \mathrm{a}, \mathrm{b}$ and c). Since the flow exits only through the effusion holes for this case, (i.e. $100 \%$ of spent air exit through the effusion hole). In addition to these, vortices are also formed near the closed edges.

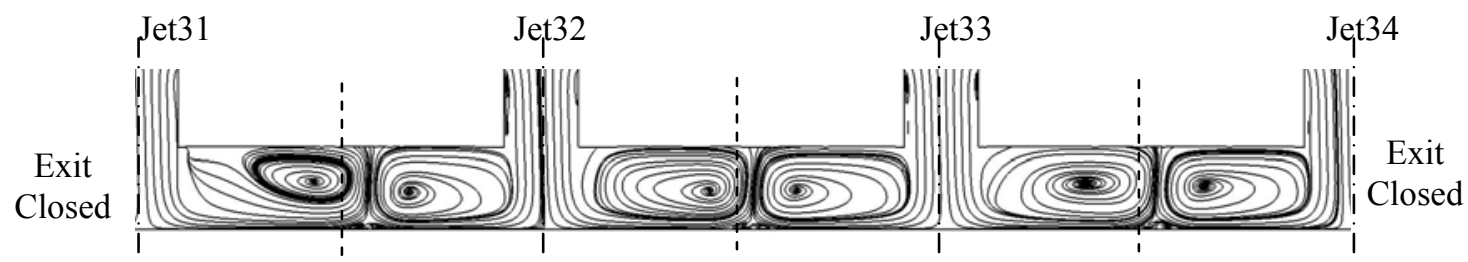

(a) plane along L1

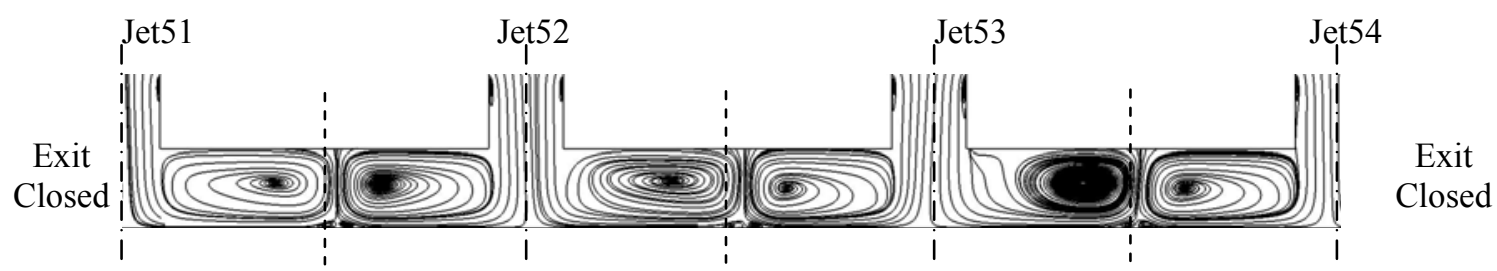

(b) plane along L2

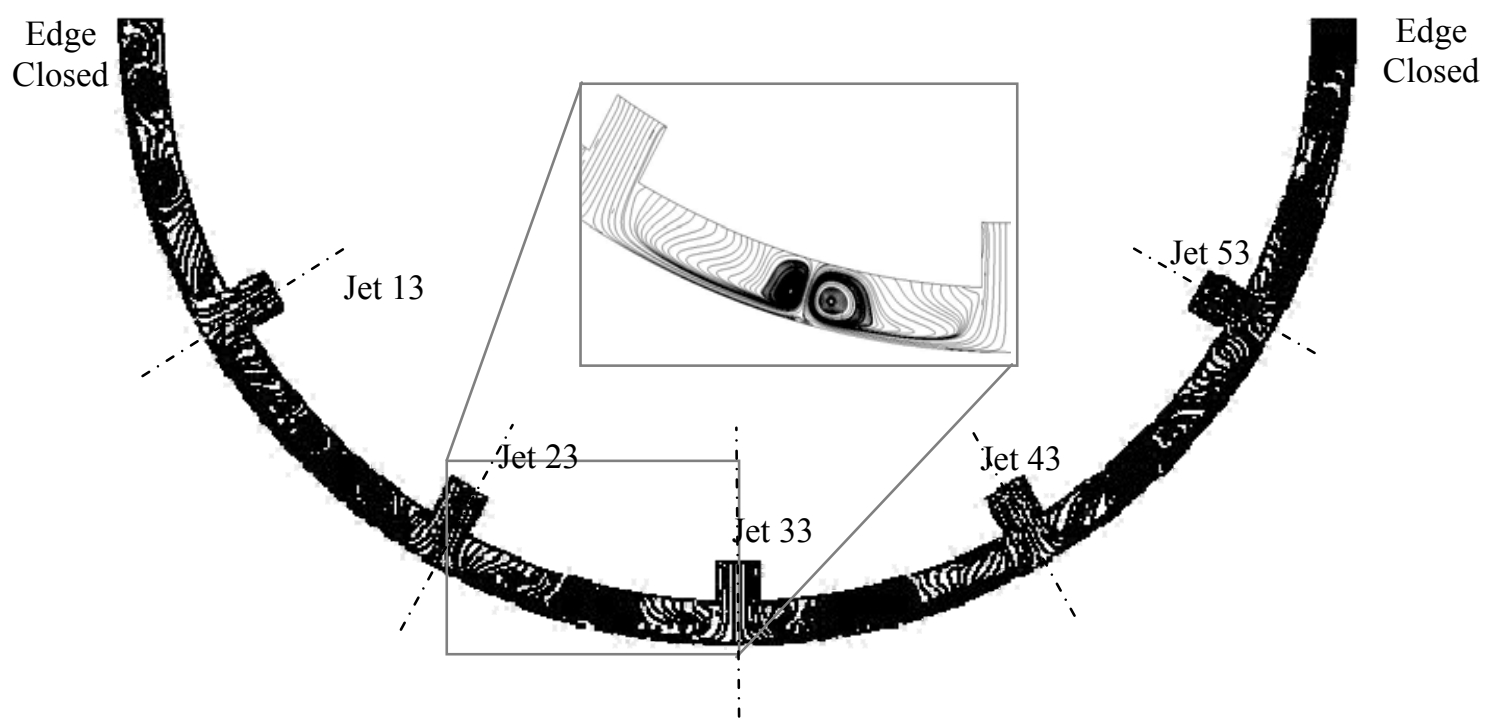

(c) along curve $\mathrm{S}$

Fig. 14 Path lines plotted for Case-Ds

\subsubsection{Exit configuration ' $D$ 'with inline hole arrangement (Case - Di)}

For inline arrangement, Case-Di (Fig. 15), the path lines plotted along the plane ' $\mathrm{S}$ ' show that eddies are not formed in the effusion holes, as the flow effuses through the effusion holes. It indicates that the suction created will be more for this exit configuration which leads to reduction in the internal cross flow. 


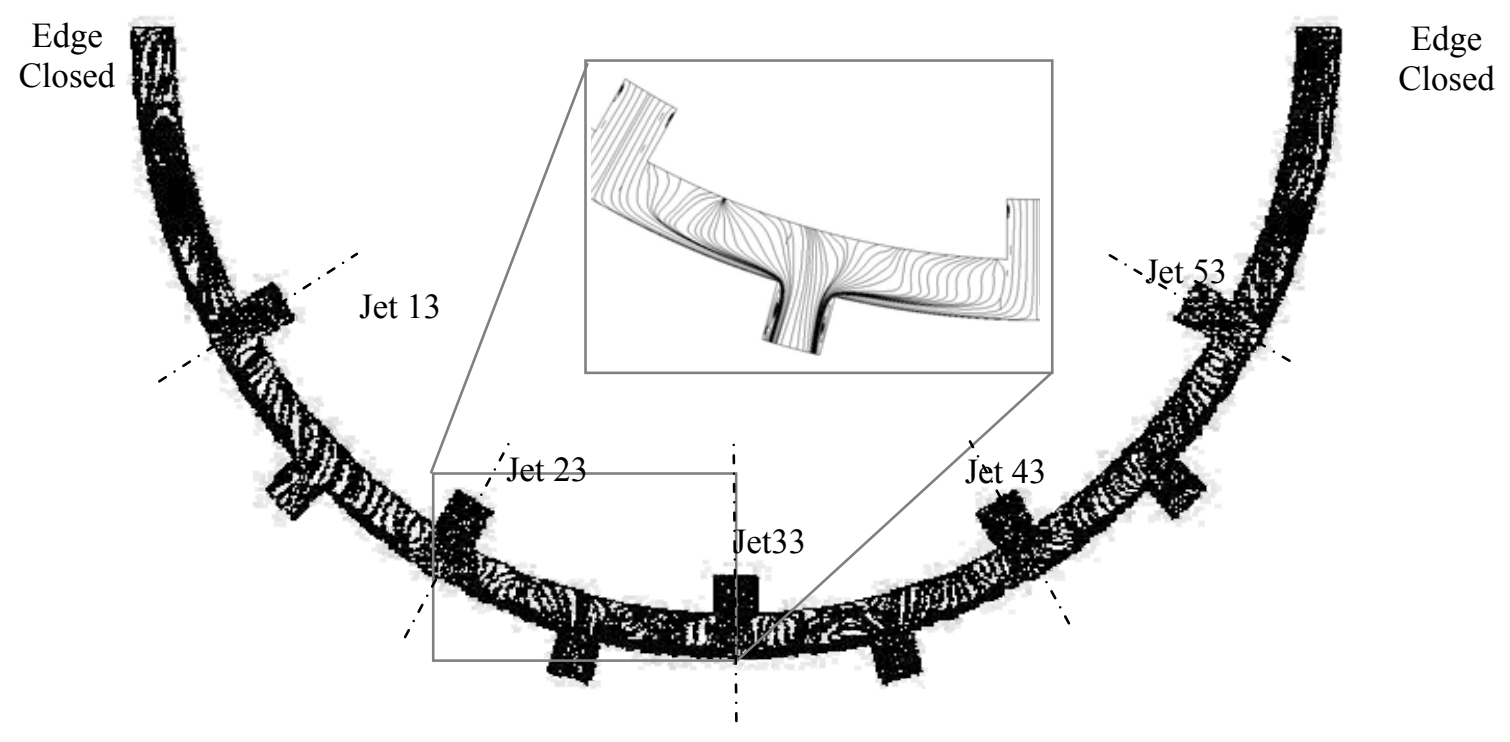

Fig. 15 Path lines plotted for Case-Di

\subsection{Pressure distribution}

Figure 16 shows the contours of static pressure on the entire target surface for different cases. Among all the four exit configurations, the Case-Ds has the higher and more uniform pressure distribution on the target surface. Case-As and Case-Bs shows symmetric pressure distributions with lower peaks. Case-Cs, on the other hand, has asymmetric pressure distribution as one edge alone is open. Similar trends are observed for inline jets also.

In order to study the effect of arrangement of effusion holes and exit configuration in detail, the dimensionless pressure coefficient $\left(\mathrm{P}^{*}\right)$ is plotted along the selected lines 'L1' and 'L2' and curve ' $\mathrm{S}$ '. They are explained in the following.

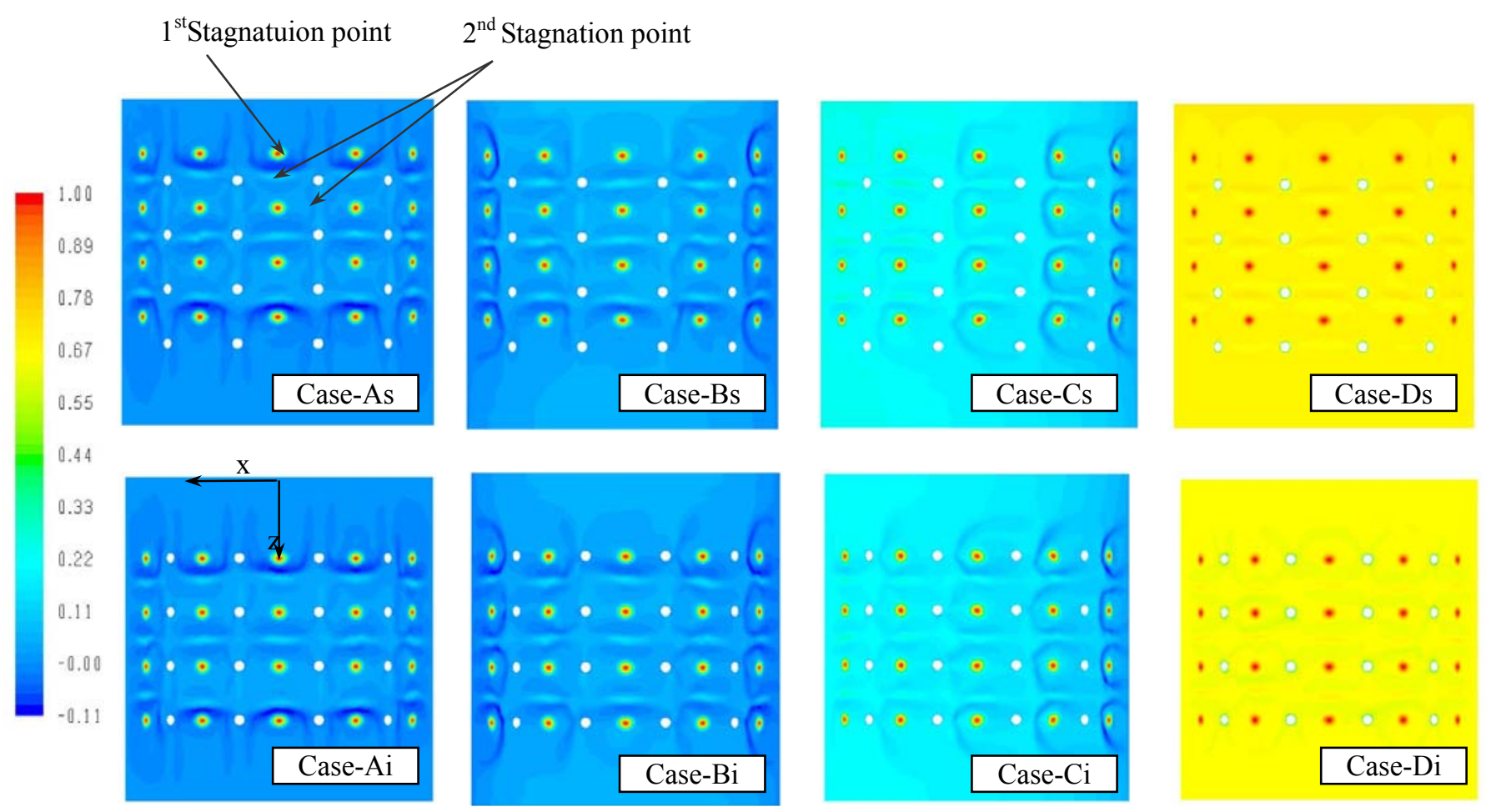

Fig. 16 Dimensionless pressure $\mathrm{P}^{*}$ contour on the target surface 


\subsubsection{Staggered effusion holes}

Consider the pressure distribution for Case-As where jet-32 interacts with jet33, Fig. 17. The value of $\mathrm{P}^{*}$ is unity at primary stagnation point and decreases as the flow accelerates from the stagnation point. In the wall jet region, the flow gets decelerated and boundary layer is formed. The $\mathrm{P}^{*}$ is almost constant in the wall jet zone, $\mathrm{z} / \mathrm{D}=11.6$ to 13.4 . A second peak in $\mathrm{P}^{*}$ with magnitude of $11 \%$ of its primary stagnation value is observed at the secondary stagnation point $\mathrm{z} / \mathrm{D}=13.5$, due to formation of secondary vortices in the up-wash region. As the flow exits through all the edges and effusion holes (Case-As), cross flow is generated in the longitudinal direction. Because of this, $\mathrm{P}^{*}$ is lower in the wall jet region than the other two exit configurations (refer Fig. 18(a)) and the $2^{\text {nd }}$ peak in $\mathrm{P}^{*}$ has shifted towards the longitudinal edges E3 and E4. For Case-Bs, edges E3 and E4 are closed and hence, the magnitude of secondary peak increases to $16 \%$ of the primary peak values. The $2^{\text {nd }}$ peak occurs between the primary peaks of adjacent jets. For Case-Cs, $\mathrm{P}^{*}$ increases in the wall jet region and the $2^{\text {nd }}$ peak increases to $22 \%$ of the primary peak values. Highest pressure values on the target surface are noticed for the Case-Ds where the air exits only through effusion holes. The pressure distribution (P*) when plotted along line 'L2' in Fig. 18(b) is similar to the one along 'L1' (Fig 18(a)) as far as the primary stagnation zone is concerned. However the secondary peak is sharper for Cases-As, Bs and Ds and rounded for cases Cs. The difference among the cases As, Bs and Cs is less pronounced along 'L2'. For case Ds, there is practically no difference between $\mathrm{P}^{*}$ values along 'L1' and 'L2'.

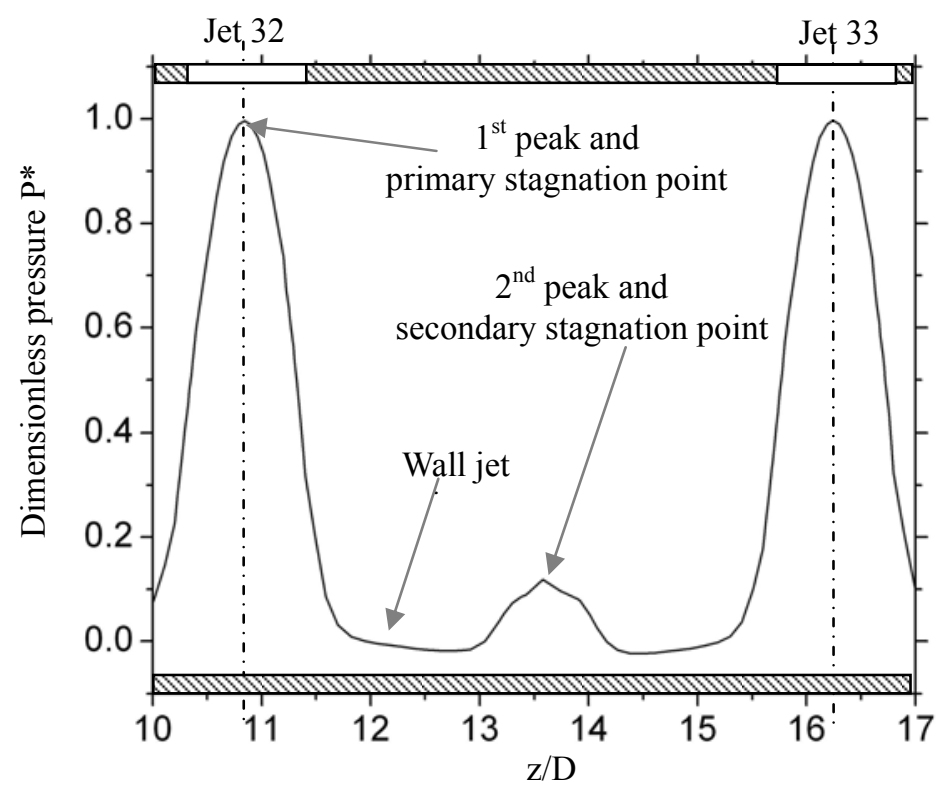

Fig. 17 Dimensionless pressure $\left(\mathrm{P}^{*}\right)$ distribution for jet32 interacting with jet33 along line L1 for Case-As

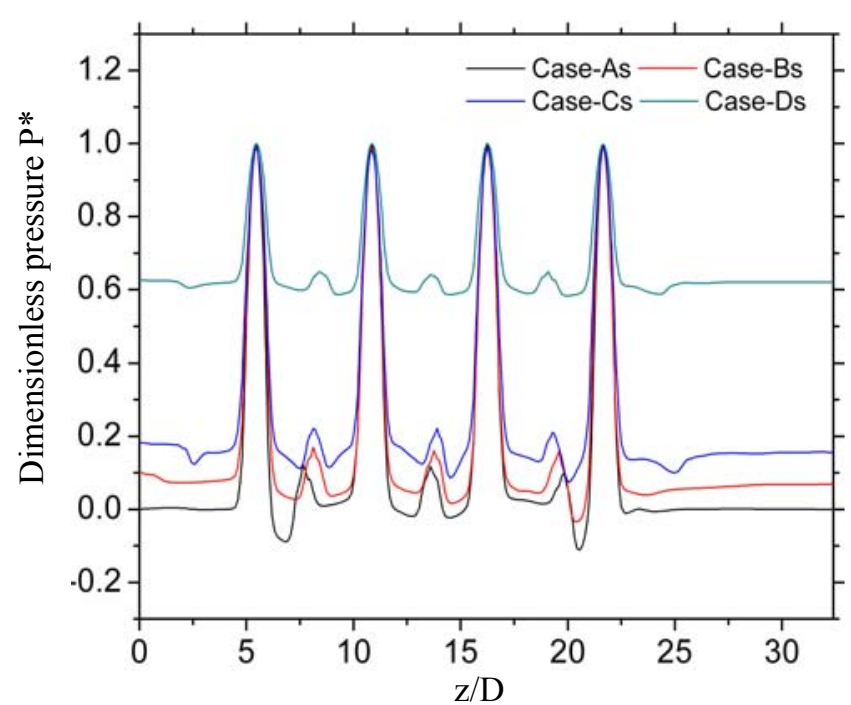

(a) $\mathrm{P} *$ distribution along $\mathrm{L} 1$

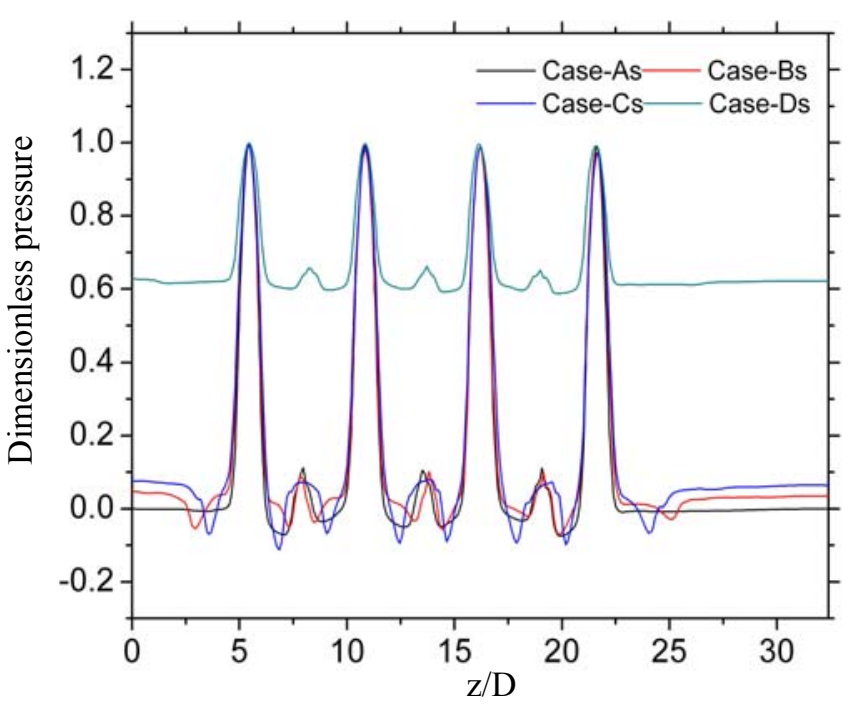

(b) $\mathrm{P}^{*}$ distribution along L2

Fig. 18 Dimensionless pressure $\mathrm{P}^{*}$ distribution along L1 and L2 for staggered effusion holes 
Figure 19 shows the $\mathrm{P} *$ distribution plotted along the curvature ' $\mathrm{S}$ ' for staggered effusion holes. For Cases As, Bs and Ds, the $\mathrm{P}$ * distributions are symmetric about the axis of jet33. For Case-Bs, It is observed that the jet interaction is observed for the center jet. Near jet31 and jet35 no pressure peaks are formed since there is no existence of PV and SV cells from the path lines. For Case-Cs, asymmetry in pressure distribution is observed. This asymmetry in pressure distribution for this configuration is due to opening of only one edge (E1), and the flow is biased towards the open edge.

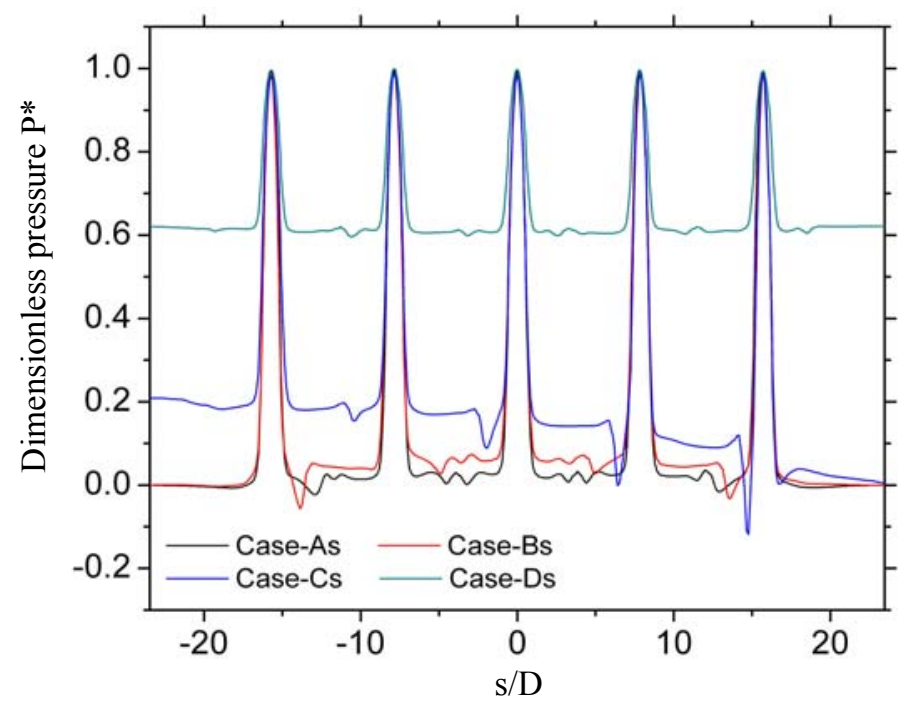

Fig. 19 Dimensionless pressure $\mathrm{P}^{*}$ distribution along $\mathrm{S}$ for staggered jets

\subsubsection{Inline effusion holes}

Figures 20 (a) and (b) show the P* distribution along the lines 'L1' and 'L2' respectively for inline jets. Along 'L1' (Fig. 20 (a)), the values of second peaks for $\mathrm{P}^{*}$ for the cases-As, Bs, Cs and Ds are 12\%, 15\%, 20\%, and 64\% of their corresponding first peak values. When compared with staggered cases, the secondary peak is reduced for exit configuration cases- $\mathrm{B}$ and $\mathrm{C}$ for inline arrangement. This reduction in pressure is due to increase in spent air exiting through the effusion holes (refer Table 1) and consequent reduction in cross flow.

Figure 20 (b) shows the $\mathrm{P}^{*}$ distribution along the line L-2 for inline arrangement. It is noticed that the distribution curve for Case-Ai, Bi and $\mathrm{Ci}$ appears to be similar to that of staggered effusion holes. However the rounding of in secondary pressure peak is not observed due to further reduction in cross flow for inline arrangement.

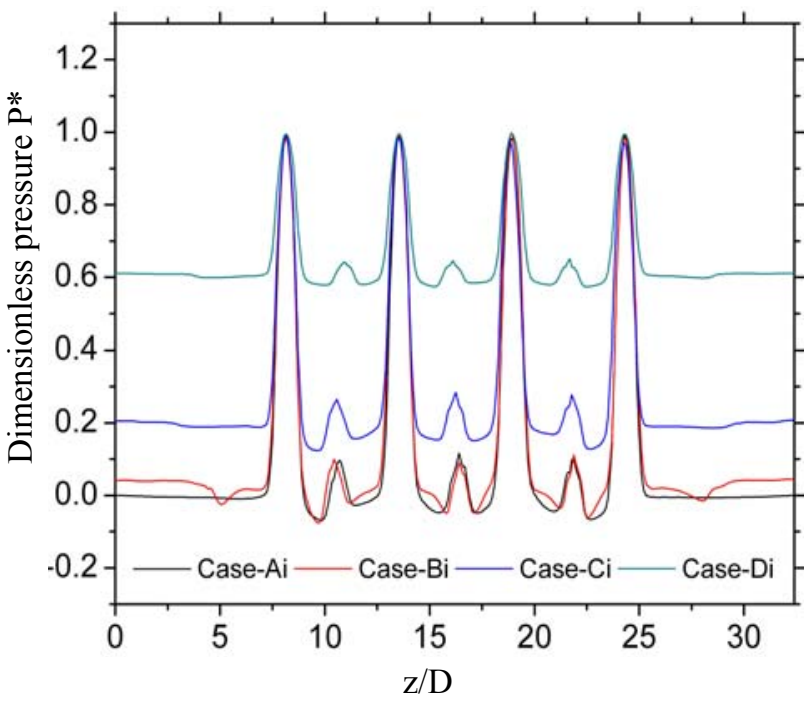

(a) $\mathrm{P} *$ distribution along $\mathrm{L} 1$

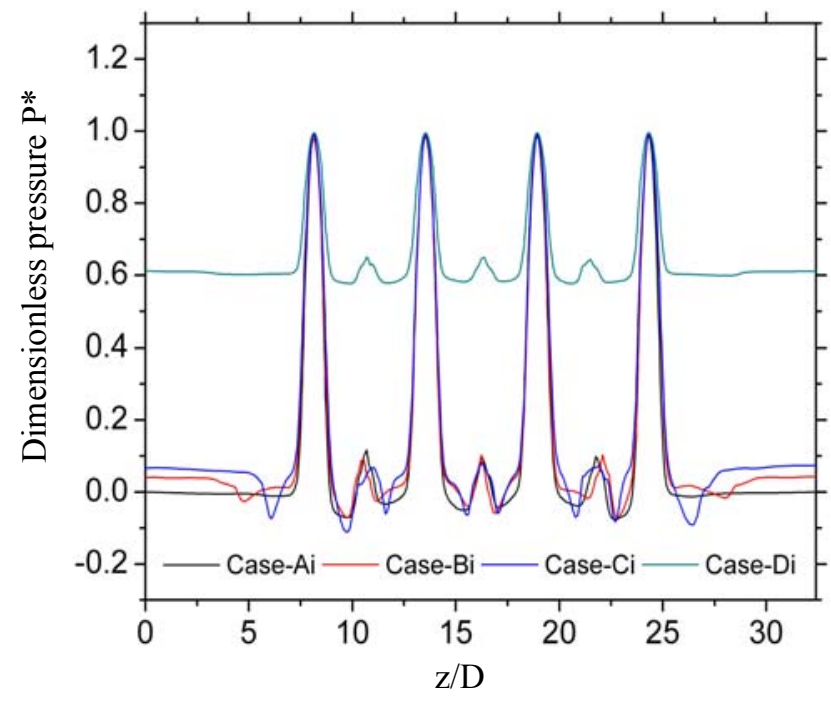

(b) $\mathrm{P}^{*}$ distribution along L2

Fig. 20 Dimensionless pressure $\mathrm{P}^{*}$ distribution along L1 and L2 for inline effusion holes 
Figure 21 shows $\mathrm{P}^{*}$ distribution along $\mathrm{S}$ for inline arrangement. The small peaks found between the first peaks noticed in the inline arrangement since the film hole partially occupies the jet interaction zone. A negative pressure is created near the edges of effusion holes. For Case-Di, the negative pressure created is relatively higher because the suction created is larger as the flow exits only through the effusion holes.

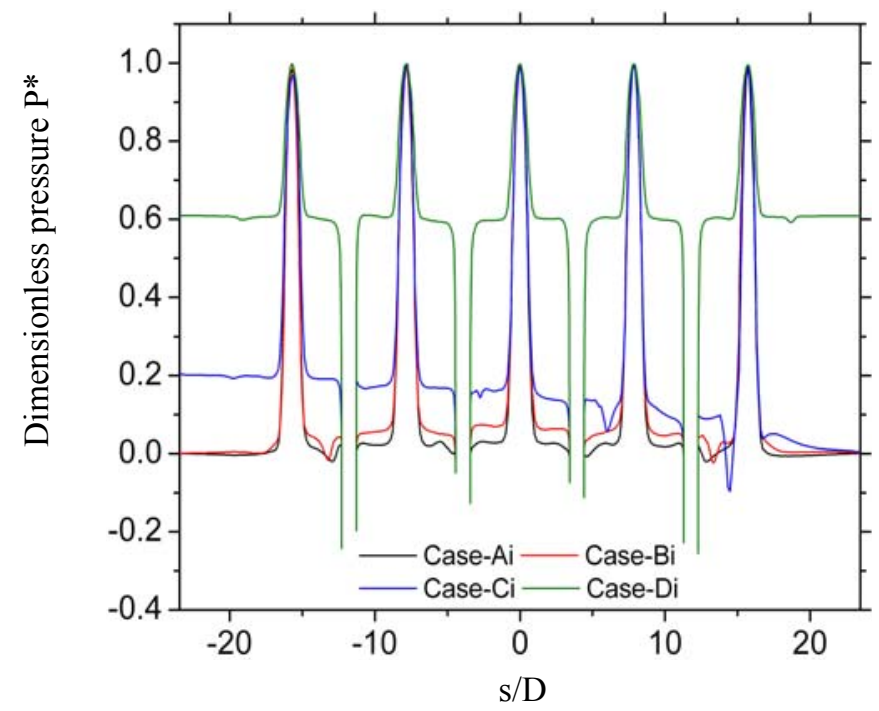

Fig. 21 Dimensionless pressure $\mathrm{P} *$ distribution along $\mathrm{S}$ for inline jets

\subsection{Heat transfer characteristics}

Figure 22 shows the contour of Nusselt number on the entire target surface. In general, the heat transfer is higher at the stagnation zone and lower in the wall jet region. Higher heat transfer region is noticed in between nonbearing stagnation zones. It is observed that among all the four exit configurations, the Case-Cs, has asymmetric heat transfer distribution as one edge is open.

In order to study the effect of arrangement of effusion holes and exit configuration in detail, the Nusselt number is plotted along the lines and curves such as 'L1', 'L2' and 'S' respectively and explained in the following.

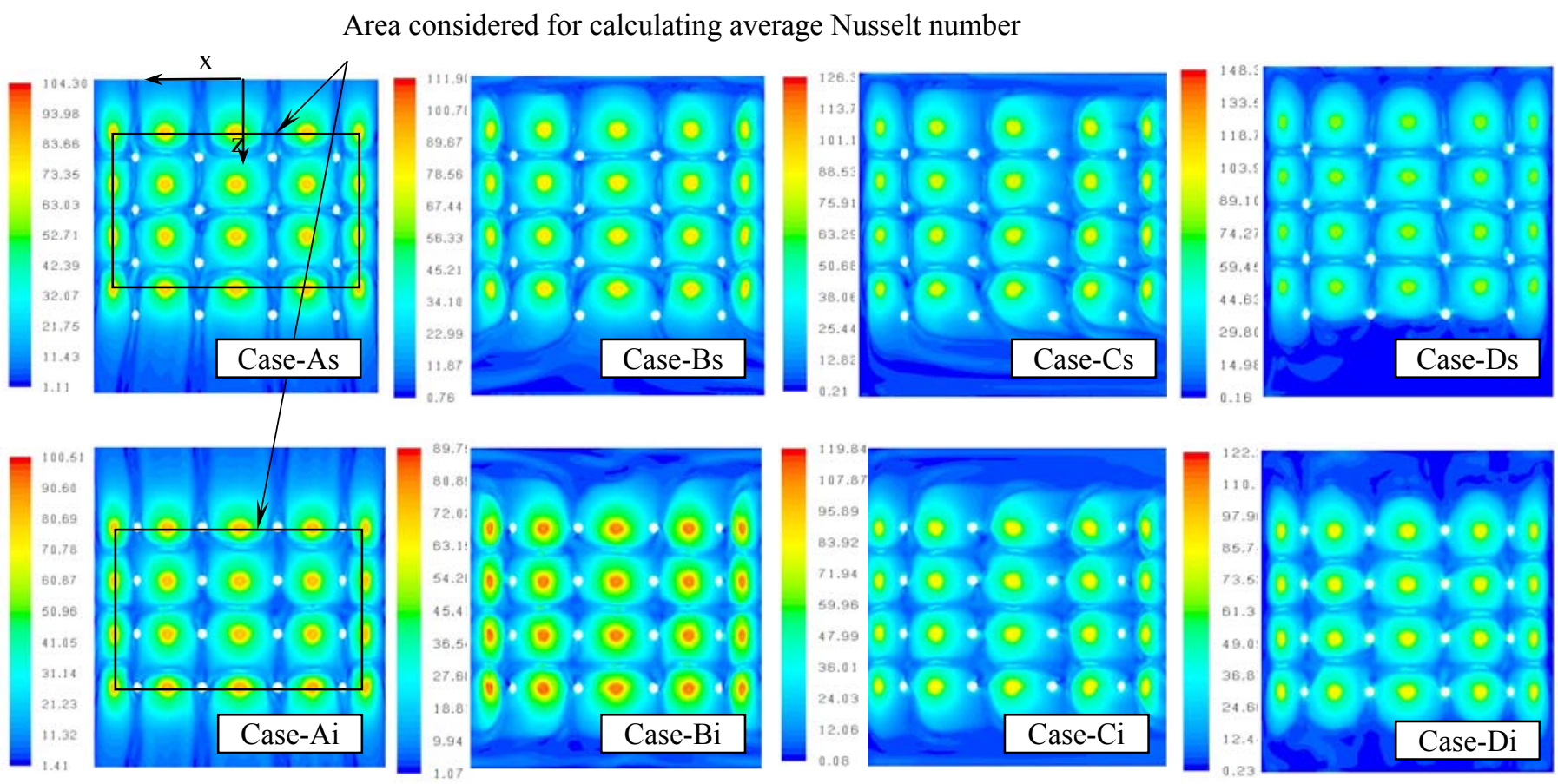

Fig. 22 Nusselt number contour on the target surface 


\subsubsection{Staggered effusion holes}

Consider the distributions of normalized Nusselt number for Case-As where jet-32 interacts with jet-33, Fig 23 (a). The Nusselt number slightly increases from the primary stagnation point $(x / D=10.8)$ till it reaches the first peak at a distance approximately equal to the radius of the nozzle. The first peak in Nusselt number is about $7 \%$ larger than the stagnation value this increase is due to the increase pressure and velocity gradients, which in thru gives raise to increase in turbulence production around the nozzle edge, refer Fig. 23 (b). Figure 23(a) also shows the corresponding pressure distribution, reproduced from Fig. 17. It is evident that the Nusselt number reduces from the first peak due to formation of boundary layer in the wall jet region. In the secondary stagnation zone, the Nusselt number first reduces to the lowest value and again reaches a secondary peak at the secondary stagnation point $(\mathrm{x} / \mathrm{D}=13.7)$ with a magnitude of about $45 \%$ of the primary stagnation point value. Thus the second peak is obviously due to formation of secondary stagnation zone in the up-wash region. Figures 24 (a) and (b) show the effect of exit closure on Nusselt number distribution along the stagnation lines 'L1' and 'L2' for staggered effusion holes. It is observed from Fig. 24 (a) that, for Case-As, the Nusselt number distribution appears almost symmetric about the stagnation point for all the jets.

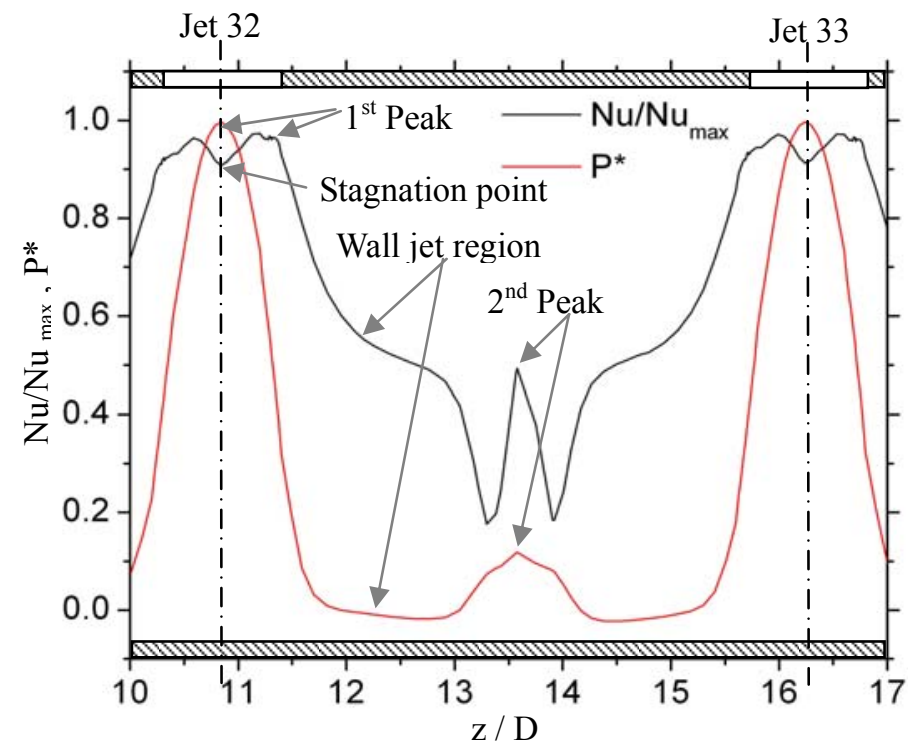

(a) Nusselt number distribution for jet32 interacting with jet33 along line L1 for Case-As

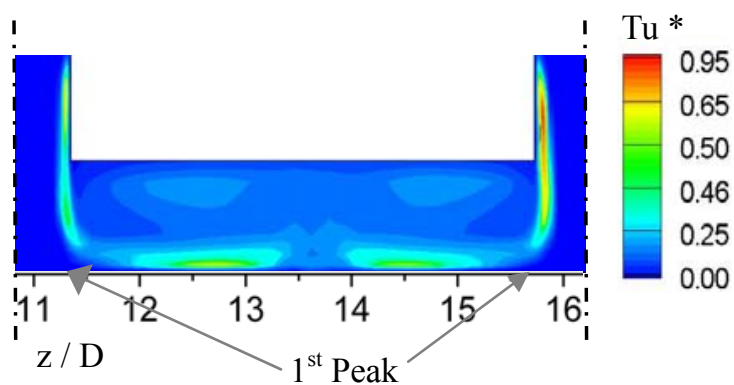

(b)Turbulent kinetic energy for jet32 interacting with jet33 along line L1 for Case-As

Fig. 23 for jet32 interacting with jet33 on line and plane along L1 for an exit configuration with staggered effusion holes with all edges open (Case-As)

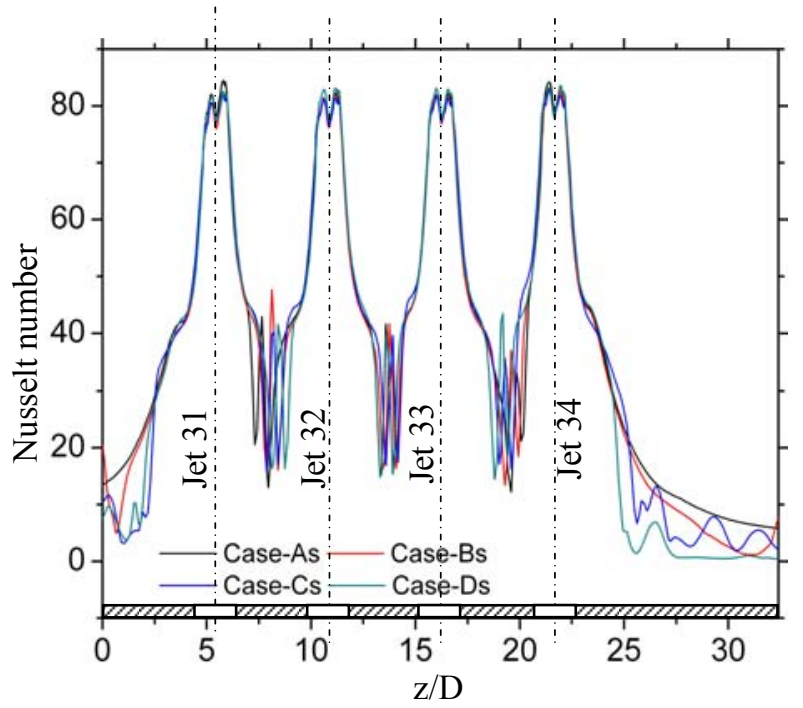

(a) Nusselt number distribution along L1

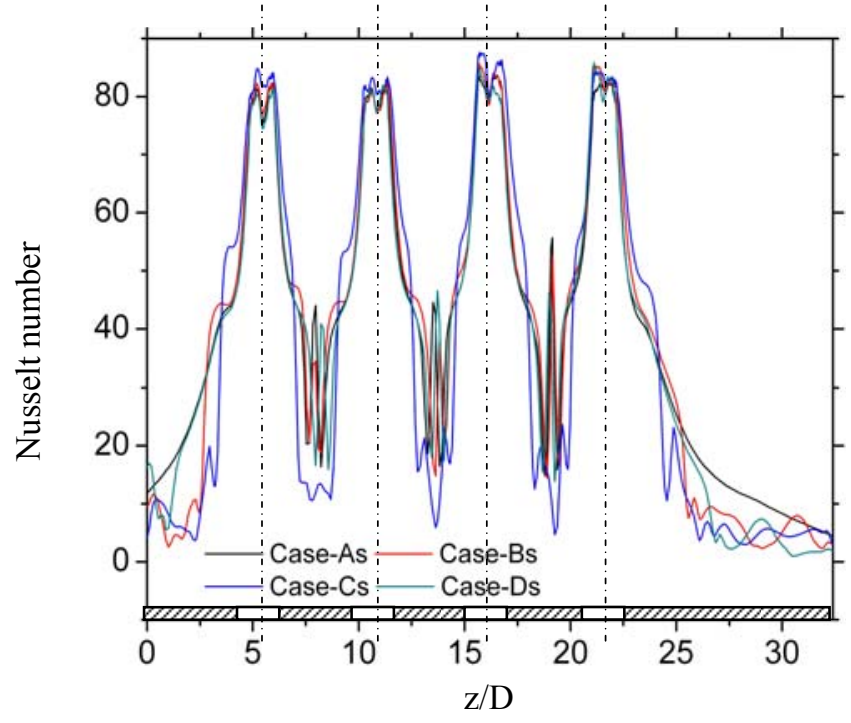

(b) Nusselt number distribution along L2

Fig. 24 Nusselt number distribution along L1 and L2 for staggered effusion holes 
The trends of the Nusselt number distributions along 'L1' are almost similar for all the exit configurations. However, with resemblance of flow characteristics, for Case-As, the second peak in Nusselt number for jet 31 and 34 are shifted away towards the open edge and the flat distribution of Nusselt number in the wall jet region is not seen. Along 'L2' (Fig. 24(b)), the Nusselt number is not much affected in the stagnation region for all cases. For Case-Cs, the Nusselt number is slightly higher in the wall jet region and the secondary peak in Nusselt number completely disappeared. This behavior is due to the wash away cross flow which destroys the formation of secondary vortices.

Figure 25 shows the Nusselt number distribution along the curve ' $\mathrm{S}$ ' for all the exit configurations. Due to weakening of the secondary vortices and second peak in pressure along the curvature ' $S$ ' as discussed in previous section, the second peak in Nusselt number is small along ' $\mathrm{S}$ ' compared to that of line ' $\mathrm{L} 1$ '. The Nusselt number distribution exhibits symmetry about $\mathrm{s} / \mathrm{D}=0$, However for Case-Cs, Nusselt number distribution is asymmetric due to increase in cross flow along ' $\mathrm{S}$ '. In addition, it is noted that the location of second peak has shifted towards the opening edge and the magnitude of second peak is increases from jet13 to jet53. For Case-Cs, the first peak increases along positive s/D due to increase in convection heat transfer.

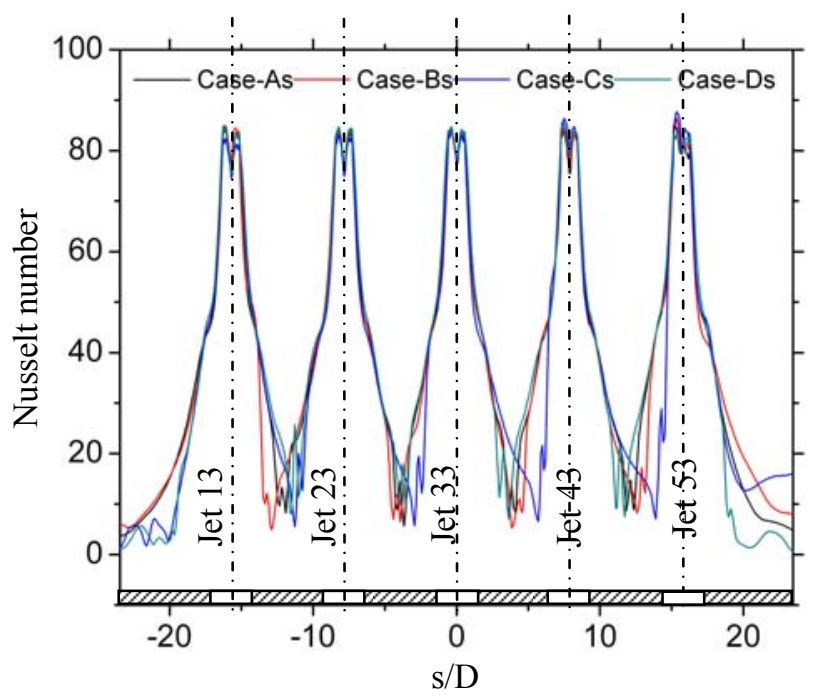

Fig. 25 Nusselt number distribution along S for staggered effusion holes

\subsubsection{Inline effusion holes}

Figures 26 (a) and (b) show the Nusselt number values along 'L1' and 'L2' for inline effusion holes. For Case-Ci, Along 'L1', (Fig. 26(a)) the heat transfer characteristics are almost similar to that of staggered effusion holes. However, along 'L2', (Fig. 26(b) the secondary peak in Nusselt number is not completely washed away and there exist second peak in Nusselt number with small magnitude. It is due to reduction on internal cross flow when the effusion holes are inline with jet holes.

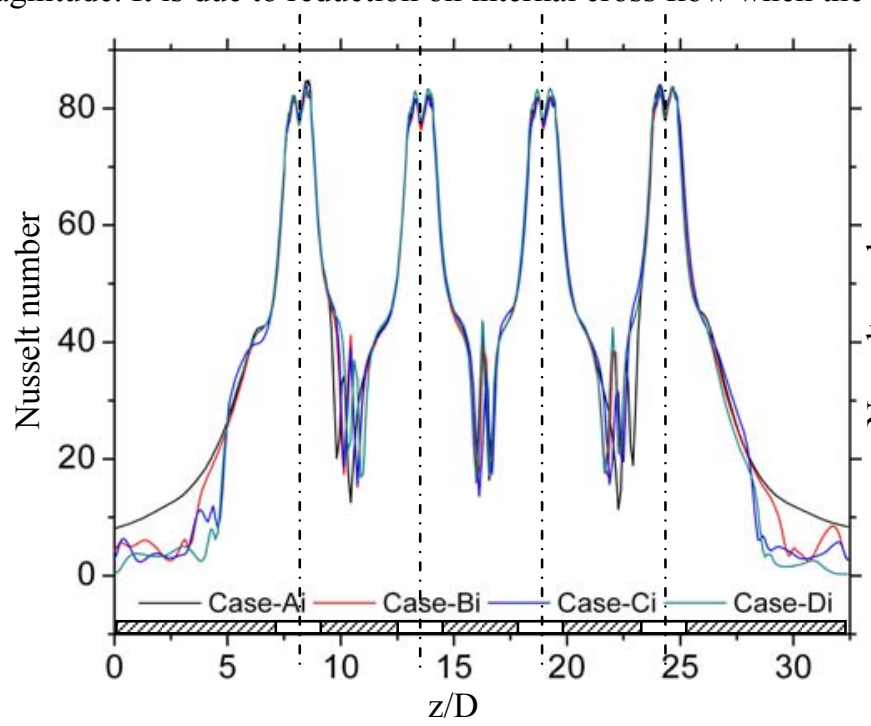

(a) Nusselt number distribution along L1

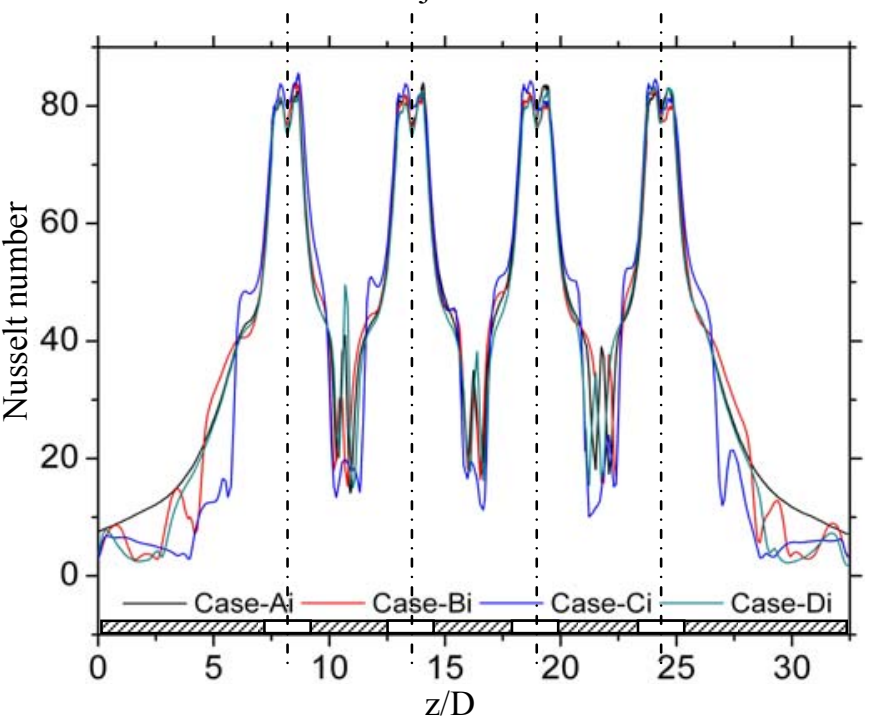

(b) Nusselt number distribution along L2

Fig. 26 Nusselt number distribution along L1 and L2 for inline effusion holes 
Figure 27 shows the Nusselt number distribution along the curve S for inline effusion holes. Sharp increase in heat transfer near the edges of effusion holes are identified in Fig. 27. This increase in heat transfer near the edges of effusion holes are higher for Case-Di due to the suction created near the edges of effusion holes.

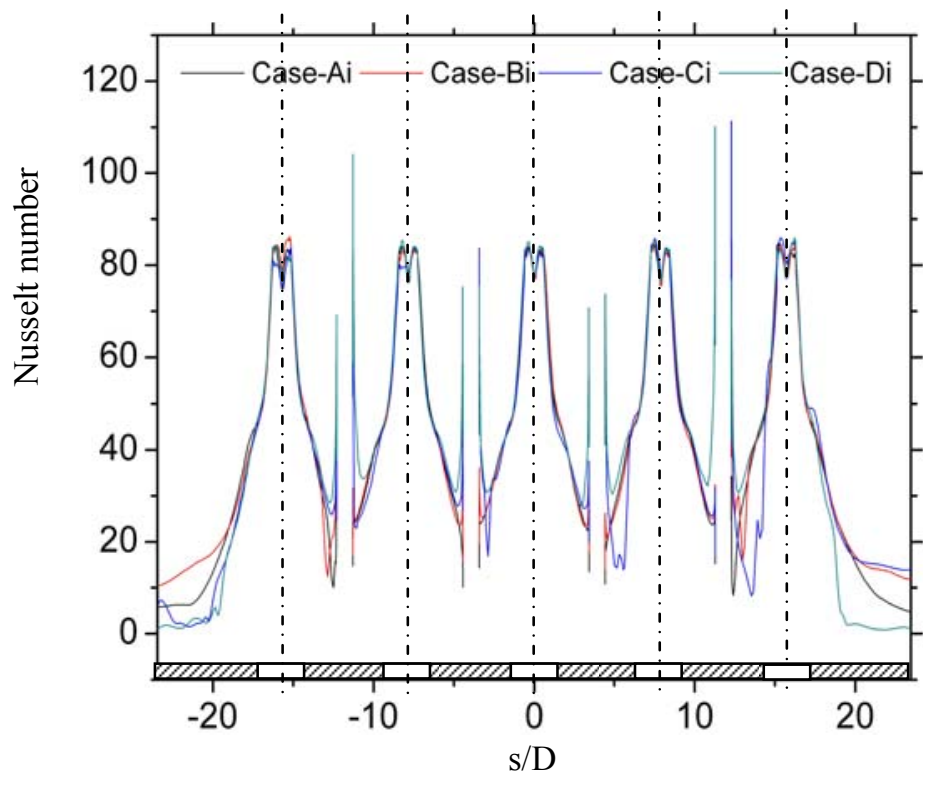

Fig. 27 Nusselt number distribution along $\mathrm{S}$ for inline effusion holes

\subsubsection{Average Nusselt number}

In order to find the effect of exit closure in heat transfer the local Nusselt number is averaged in the specified area (refer Fig. 22). Figure 28 shows the effect of hole arrangements and exit configurations on average heat transfer. It is observed that when flow exits only through staggered effusion holes heat transfer values are higher. The configuration with flow exit through staggered effusion holes and along one edge will have lower heat transfer value. The discrepancies in average heat transfer values are attributed to the percent of spent air exit through the effusion holes. Heat transfer is higher when the percent of spent air exit through the effusion hole is larger (i.e. internal cross flow is reduced) and vice versa.

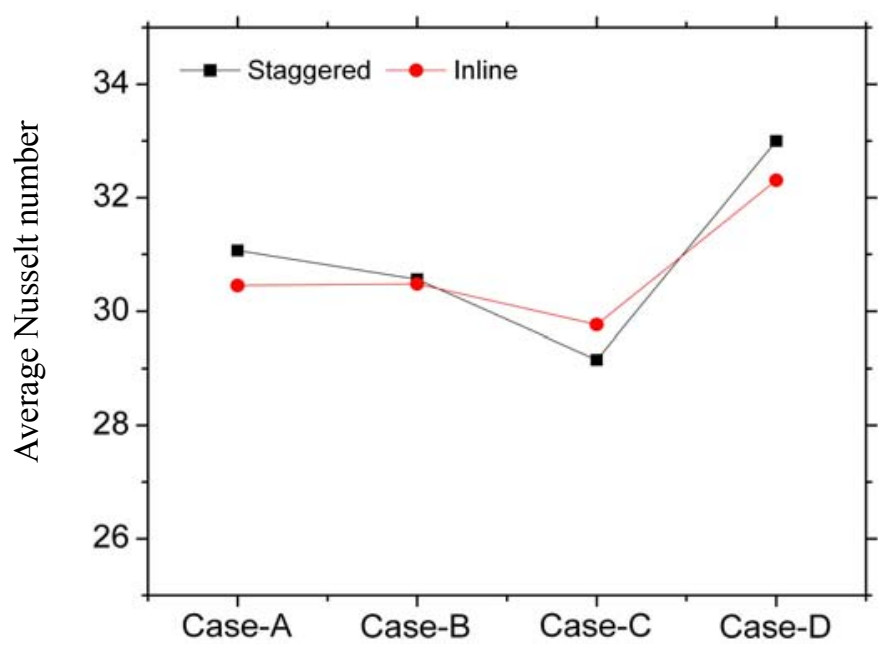

Fig. 28 Average Nusselt number for all the cases

\section{Conclusions}

Computational study is carried out to determine the flow and heat transfer characteristics for multiple rows of jets impinging on the concave target surface with multiple rows of effusion holes. Exit configurations, arrangement of holes, Effect of arrangement of jet and effusion holes for a Reynolds numbers of 7500 are investigated. The following conclusions are made from the study.

1. A good qualitative correlation is found among the flow patterns, pressure variations and heat transfer characteristics for various cases investigated.

2. The flow is characterized by primary vortex pair, up-wash and secondary vortex pair forming second stagnation point.

3. The exit configuration with one edge is open (Case-C) shows asymmetry in flow characteristic, pressure and heat transfer distributions.

4. The heat transfer is characterized by first and second peak in Nusselt number. The First peak in Nusselt number is attributed to increase in turbulence produced around the nozzle edge and the second peak is due to formation of second stagnation zone. 
5. The average heat transfer is least for the exit configuration with staggered effusion and one edge open (Case-Cs). It is due to high cross flow produced as the flow is primarily biased towards the open edge.

6. The average heat transfer is maximum for the exit configuration with all the edges closed (Case-Ds).

\section{Nomenclature}

$\begin{array}{llll}d & \text { Effusion hole diameter }[\mathrm{m}] & & \text { Greek symbols } \\ D & \text { Jet orifice diameter }[\mathrm{m}] & \kappa & \text { Turbulent kinetic energy }\left[\mathrm{m}^{2} / \mathrm{s}^{2}\right] \\ P & \text { Pressure }\left[\mathrm{N} / \mathrm{m}^{2}\right] & \mu & \text { Dynamic viscosity }[\mathrm{kg} / \mathrm{m}-\mathrm{s}] \\ P^{*} & \text { Dimensionless pressure } & & \text { Kinematic viscosity }\left[\mathrm{m}^{2} / \mathrm{s}\right] \\ H & \text { Jet hole to target surface distance (height) }[\mathrm{m}] & & \text { Specific dissipation rate }[1 / \mathrm{s}] \\ T & \text { Temperature }[\mathrm{K}] & \rho & \text { Density }\left[\mathrm{kg} / \mathrm{m}^{3}\right] \\ h & \text { Heat transfer coefficient }\left[\mathrm{W} / \mathrm{m}^{2} \mathrm{~K}\right] & & \text { Gradient operator } \\ k & \text { Thermal conductivity }[\mathrm{W} / \mathrm{mK}] & & \text { Subscripts } \\ m & \text { Mass flow }[\mathrm{kg} / \mathrm{s}] & & \text { Flow through one hole } \\ x & \text { Length of target surface along X-coordinate }[\mathrm{m}] & 1 & \text { Total value } \\ s & \text { Length of target surface along circumference }[\mathrm{m}] & \text { total } & \text { Maximum value along line or plane considered } \\ N u & \text { Nusselt number }\left(=h_{x} D / k_{f}\right) & m a x & \text { Target plate } \\ R e & \text { Jet Reynolds number }\left(=4 m_{l} / \pi D \mu\right) & t p & \text { Wall } \\ q & \text { Heat flux }\left[\mathrm{W} / \mathrm{m}^{2}\right] & w & \text { Local value } \\ T u^{*} & \text { Dimensionless turbulent kinetic energy }(=\kappa / \kappa \text { max }) & x & f\end{array}$

TKE Abbreviations

$S D R \quad$ Turbulent kinetic energy

Specific dissipation rate

\section{Reference}

[1] Hollworth, B. R. and Dangan, L., 1980, “Arrays of Impinging Jets with spent fluid removal through vent holes on the target surface - Part I: Average Heat Transfer," Transactions of the ASME, Journal of Engineering for power, Vol. 102, pp. 994-999.

[2] Hollworth, B. R., Lehmann, G., and Rosiczkowski J., 1983, “Arrays of Impinging Jets with spent fluid removal through vent holes on the target surface - Part II: Local Heat Transfer," Transactions of the ASME, Journal of Engineering for Power, Vol. 105, pp. 393-402.

[3] Cho, H. H. and Rhee, D. H., 2001, "Local Heat/Mass Transfer Measurement on the Effusion Plate in Impingement/Effusion Cooling Systems," Journal of Turbomachinery, Vol. 123, pp. 601-608.

[4] Ekkad, S. V., Huang, Y. and Han J. C., 1999, "Impingement Heat Transfer on a Target Plate with Film cooling Holes,” AIAA Journal of Thermophysics and Heat Transfer, Vol. 13, No. 4, pp. 522-528.

[5] Rhee, D. H., Choi, J. H., and Cho H. H., 2003, "Flow and Heat transfer characteristics in an Impingement/ Effusion cooling system with cross flow," Transaction of ASME Journal of Turbomachinery, Vol. 125, pp. 74-82.

[6] Rhee, D. H., Yoon, P. H., and Cho, H. H., 2003, "Local heat/mass transfer and flow characteristics of array impinging jets with effusion holes ejecting spent air," International Journal of Heat and Mass Transfer, Vol. 46, pp. 1049-1061.

[7] Metzger, D.E., Yamashita, T., and Jenkins, C. W., 1969, "Impingement cooling of concave surfaces with lines of circular air jets," Transaction of ASME Journal of Engineering for Power, July, pp. 149-158.

[8] Thomann, H., 1968, "Effect of stream wise wall curvature on heat transfer in a turbulent boundary layer," J. Fluid Mech., Vol. 33, No. 2, pp.283-292.

[9] Tabakoff, W., and Clevenger, W., 1972, "Gas Turbine Blade Heat Transfer Augmentation by Impingement of Air jets Having Various Configurations," Transaction of ASME Journal of Engineering for Power, Jan, pp. 51-60.

[10]Bunker, R.S., and Metzger, D.E., 1990, "Local Heat Transfer in Internally Cooled Turbine Airfoil Leading Edge Regions: Part I-Impingement Cooling Without Film Coolant Extraction," Transactions of the ASME Journal of Turbomachinery, Vol. 112, pp. 451-458.

[11]Bunker, R.S., and Metzger, D.E., 1990, "Local Heat Transfer in Internally Cooled Turbine Airfoil Leading Edge Regions: Part II-Impingement Cooling With Film Coolant Extraction,” Transactions of the ASME Journal of Turbomachinery, Vol. 112, pp. 459467.

[12]Taslim, M. E., and Khanicheh, A., 2006, "Experimental and Numerical study of Impingement on an Airfoil Leading edge with and without shower headed and gill film holes," Transaction of ASME Journal of Turbomachinery, Vol. 128, pp. 310-320.

[13] Ramakumar, B. V. N., and Prasad, B. V. S. S. S., 2006, "Computational investigation of flow and heat transfer for a row of circular jets impinging on a concave surface,” ASME Turbo Expo, GT 2006-90851.

[14] Ramakumar, B. V. N., and Prasad, B. V. S. S. S., 2008, "Computational flow and heat transfer of a row of circular jets impinging on a concave surface," Heat and Mass Transfer, Vol. 44, No. 6, pp. 667- 678.

[15] Ashok Kumar, M., and Prasad, B.V.S.S.S., 2011, "Computational flow and heat transfer of multiple circular jets impinging on a flat surface with effusion holes," Heat Mass Transfer, Vol. 47, pp.1121-1132.

[16] Lee, D. H., Chung, Y.S., and Won, S.Y., 1999, "The effect of concave surface on heat transfer from a fully developed round impinging jet," International journal of Heat and Mass Transfer, Vol. 42, No. 13, pp. 2489-2497. 